Light Water Reactor Sustainability Program

Human Factors Engineering Insights and Guidance for Implementing Innovative Technologies from

the Nuclear Innovation Workshop: A Summary Report

September 2019

U.S. Department of Energy

Office of Nuclear Energy 


\section{Disclaimer}

This information was prepared as an account of work sponsored by an agency of the U.S. Government. Neither the U.S. Government nor any agency thereof, nor any of their employees, makes any warranty, expressed or implied, or assumes any legal liability or responsibility for the accuracy, completeness, or usefulness, of any information, apparatus, product, or process disclosed, or represents that its use would not infringe privately owned rights. References herein to any specific commercial product, process, or service by trade name, trade mark, manufacturer, or otherwise, does not necessarily constitute or imply its endorsement, recommendation, or favoring by the U.S. Government or any agency thereof. The views and opinions of authors expressed herein do not necessarily state or reflect those of the U.S. Government or any agency thereof. 
INL/EXT-19-55529

Revision 0

\title{
Human Factors Engineering Insights and Guidance for Implementing Innovative Technologies from the Nuclear Innovation Workshop: A Summary Report
}

\author{
Casey Kovesdi, Shawn St Germain, Katya Le Blanc, and Craig Primer
}

September 2019

Prepared for the

U.S. Department of Energy

Office of Nuclear Energy 


\section{ACKNOWLEDGEMENTS}

We would like to thank our industry partners who attended this workshop. Without their participation, the workshop would have not been possible. To this end, we are particularly grateful for the effort and support Dustin Greenwood of NuScale, Patrick Kopfle of Dominion Energy, Jim Hill of Xcel Energy, and Asgeir Drøivoldsmo of the Institute for Energy Technology provided in developing presentations to share at the workshop. Indeed, these presentations were absolutely integral to the engagement and success of the workshop.

We would also like to thank Ahmad Al Rashdan, Vaibhav Yadav, Vivek Agarwal, Michael Wheeler, Joel Corporan, Koushik Araseethota, Jeffrey Joe, Jeremy Mohon, Larry Powell, Roger Boza, Kristyn St. Clair, and Daniel Flores Jr. for all of the work that went into the design and development of key content that was distributed at the workshop and documented here. This report was made possible through funding by the United States Department of Energy Light Water Reactor Sustainability Program. Lastly, we would like to thank Alison Hahn of the Department of Energy and Ken Thomas, Craig Primer, and Bruce Hallbert of Idaho National Laboratory for championing this effort. 


\section{SUMMARY}

The Nuclear Innovation Workshop was sponsored by the United States Department of Energy Light Water Reactor Sustainability Program and held at Idaho National Laboratory on June 26-28, 2019. The objectives of this workshop were to [1] promote an innovation ecosystem that reduces cost and risk through early evaluations, [2] provide hands-on activities that demonstrate the value of using the advanced tools, methods, and capabilities offered by the Light Water Reactor Sustainability Program, [3] provide a platform to enable broad innovation in the nuclear industry, [4] enable industry leaders the opportunity for networking and sharing of their experiences, and [5] take inspiration from other industries to push the envelope for transformation in the nuclear industry.

These objectives were supported through the facilitation of detailed discussions and industry presentations within the context of a four-phased approach to nuclear innovation. Open conversation about key challenges and considerations for innovation in the nuclear industry was recorded at each of facilitated discussions, which were focused on one of four phases (see Section 3). Further, a survey was administered to attendees at the close of workshop to collect feedback on its quality and their impressions of future initiatives for promoting an innovation ecosystem (see Section 4).

Key recommendations that came from the workshop broadly included the development of an innovation portal, establishment of an innovation working group, and holding subsequent meetings with industry partners, including utilities, vendors, and research organizations to exchange lessons learned in innovation, progress on the innovation portal, and related matters specific to nuclear innovation.

Contact information from the attendees of this workshop, industry presentations, and all related materials can be found from the following LWRS Program webpage:

https://lwrs.inl.gov/Innovation in the Nuclear Industry June 2019/Home.a $\underline{\operatorname{spx}}$ 


\section{CONTENTS}

ACKNOWLEDGEMENTS

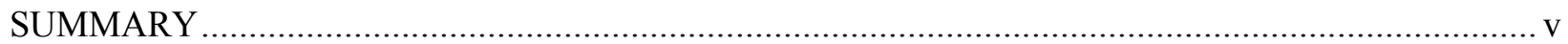

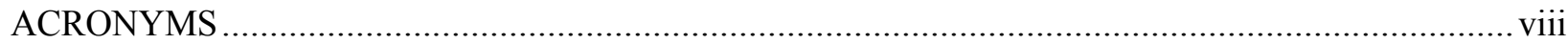

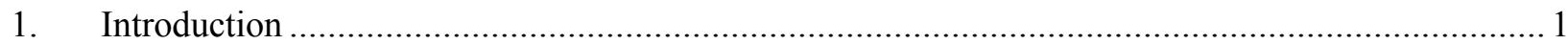

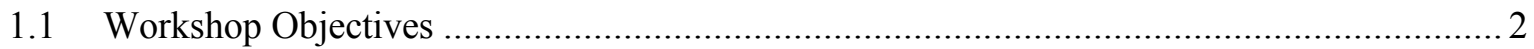

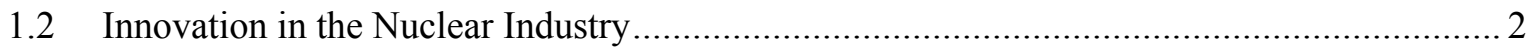

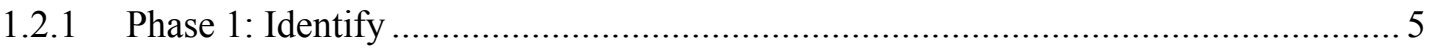

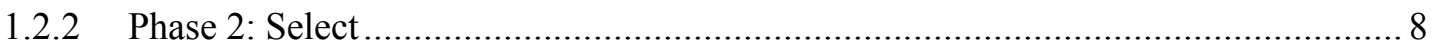

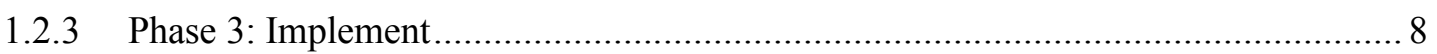

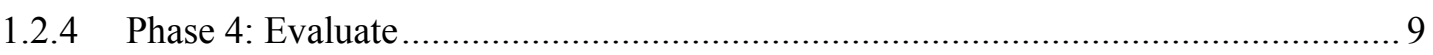

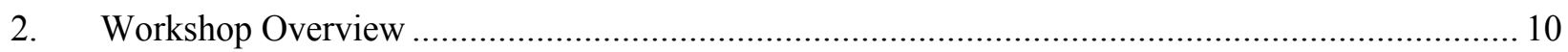

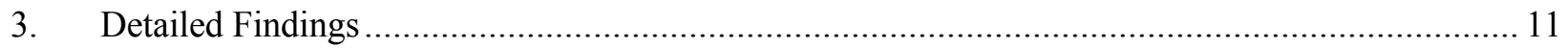

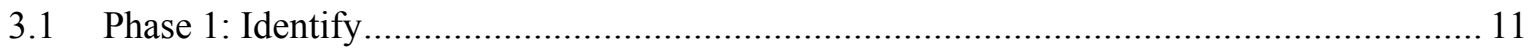

3.1.1 Presentation by Dustin Greenwood (NuScale): Identify Innovation ...................... 11

3.1.2 Facilitated Discussion 1: Identification......................................................... 12

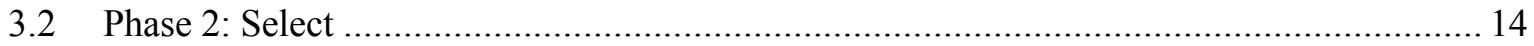

3.2.1 Presentation by Patrick Kopfle (Dominion): Nuclear Innovation Journey (Select).. 14

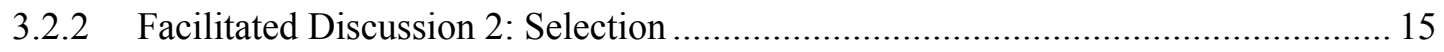

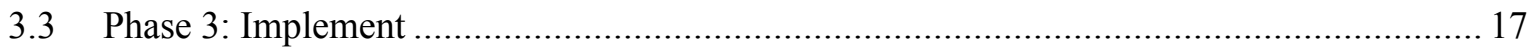

3.3.1 Presentation by James Hill (Xcel Energy): Drivers and Priorities .......................... 17

3.3.2 Facilitated Discussion 3: Implementation ...................................................... 19

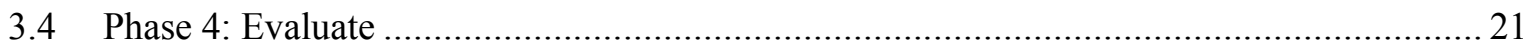

3.4.1 Presentation by Asgeir Drøivoldsmo (IFE): Evaluating Innovation Technologies -

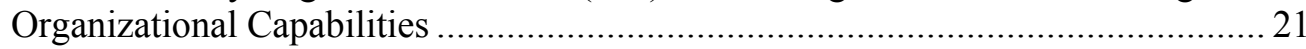

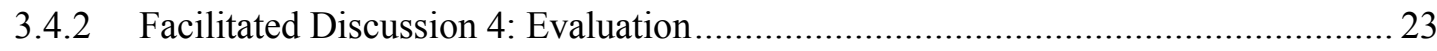

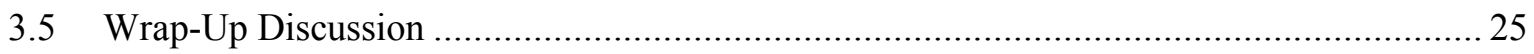

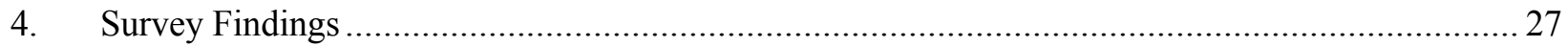

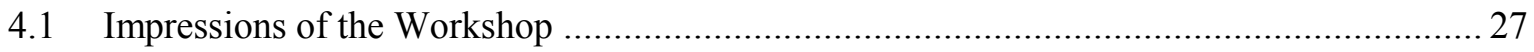

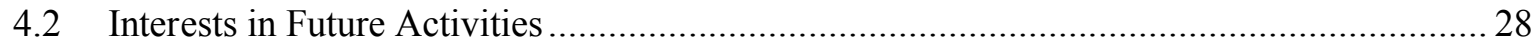

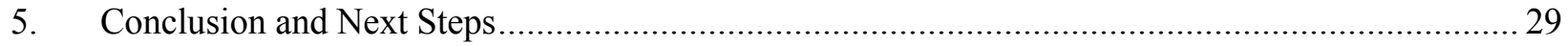

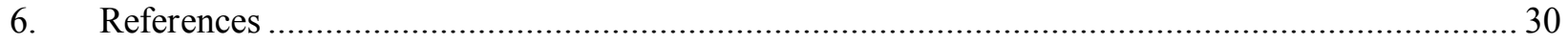

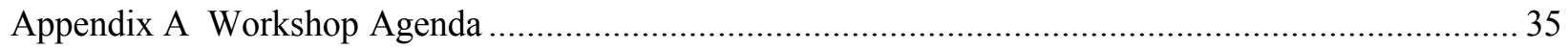

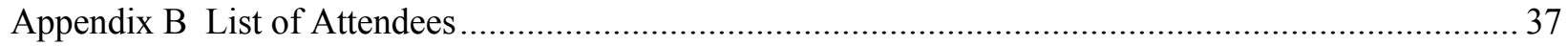

Appendix D Example Content for Base Technology Pages .......................................................... 48

Appendix E Tools Applicable to Nuclear Innovation …............................................................... 52

Appendix F Facilitated Discussion Semi-Structured Questions ...................................................... 59 


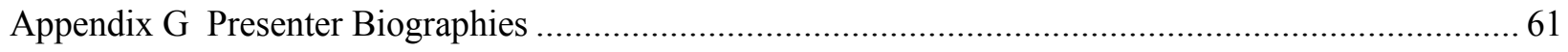

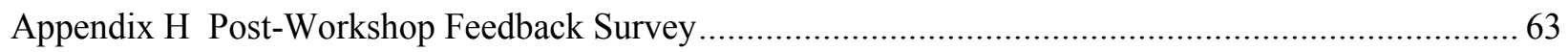

\section{FIGURES}

Figure 1. Key R\&D areas to achieve plant modernization. ............................................................. 1

Figure 2. Kotter's eight-step model for transformational change. .................................................... 3

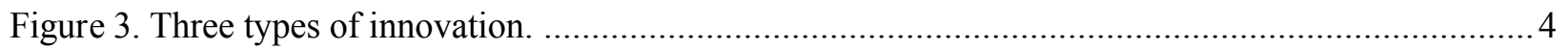

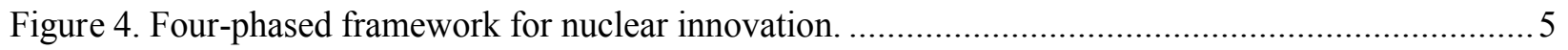

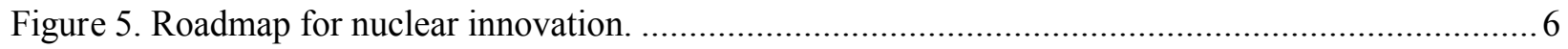

Figure 6. Example inter-relations of the base technologies, advanced capabilities, and functional

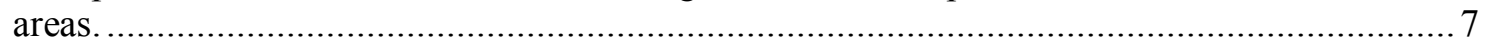

Figure 7. Human-centered design approach for nuclear innovation.................................................. 9

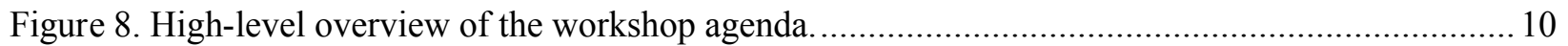

Figure 9. Identified success criteria for IO (Adopted from Asgeir Drøivoldsmo’s presentation).............21

Figure 10. Average usefulness rating of workshop with $90 \%$ confidence interval. ............................. 27

Figure 11. Frequency interested in participating in an Innovation Group if established........................28

Figure 12. Frequency interested in an Innovation Portal.............................................................28

\section{TABLES}

Table 1. Important considerations for the requirements gathering process. ........................................ 8

Table 2. Frequency count of survey completions by organization....................................................2 27 


\section{ACRONYMS}

CAVE Computer-Assisted Virtual Environment

DEG Digital Engineering Guide

DOE Department of Energy

EPRI Electric Power Research Institute

eWP Electronic Work Package

FA Function Allocation

FRA Functional Requirements Analysis

HCD Human-Centered Design

HSSL Human System Simulation Laboratory

IFE Institute for Energy Technology

INL Idaho National Laboratory

INPO Institute of Nuclear Power Operations

IO Integrated Operations

ISV Integrated System Validation

IT Information Technology

KPI Key Performance Indicator

LWR Light Water Reactor

LWRS Light Water Reactor Sustainability

MW Megawatt

$\mathrm{MHz} \quad$ Megahertz

NDA Non-Disclosure Agreement

NITSL Nuclear Information Technology Strategic Leadership

NPV Net Present Value

O\&M Operations and Maintenance

OE Operating Experience

P-CMM People Capability Maturity Model

PDF Portable Document Format

R\&D Research and Development

RFID Radio-Frequency Identification

ROI Return on Investment

SME Subject Matter Expert

SMR Small Modular Reactor

SSCs Systems, Structures, and Components 
TA Task Analysis

U.S. United States

WEC Westinghouse Electric Company

XE1 One Xcel Energy Way 


\section{Nuclear Innovation Workshop Summary Report}

\section{INTRODUCTION}

The Nuclear Innovation Workshop was sponsored by the United States (U.S.) Department of Energy's (DOE's) Light Water Reactor Sustainability (LWRS) program and held at Idaho National Laboratory (INL) on June 26-28, 2019. The overarching goal of the program is to enhance safe, efficient, and economical performance of the U.S. nuclear fleet and extend the operating lifetimes of this reliable source of electricity. This goal is addressed through two primary objectives:

1. To provide science and technology-based solutions to industry to overcome the current laborintensive business model and associated practices

2. To manage the aging of systems, structures, and components (SSCs) so nuclear power plants can continue to operate safely and cost effectively.

Program objectives are addressed through several research and development (R\&D) focus areas (i.e., pathways): Materials Research, Plant Modernization, Risk-Informed Systems Analysis, Integrated Energy Systems, and Physical Security. Specifically, the Plant Modernization pathway's strategic goal is to extend the life and improve performance of the existing U.S. nuclear power plant fleet through modernized technologies and improved processes for plant operation and power generation. The Plant Modernization pathway accomplishes this goal through demonstrating and validating new technologies and operational concepts at nuclear power plant facilities. This involves providing [1] technical-basis reports to support long-term planning, procurement, and regulatory submittals, as well as [2] guidance for full-scale implementation of modernized technologies and improved processes, communicating the results across the nuclear community. Figure 1 illustrates the Plant Modernization pathway's key areas of R\&D to achieve these goals.

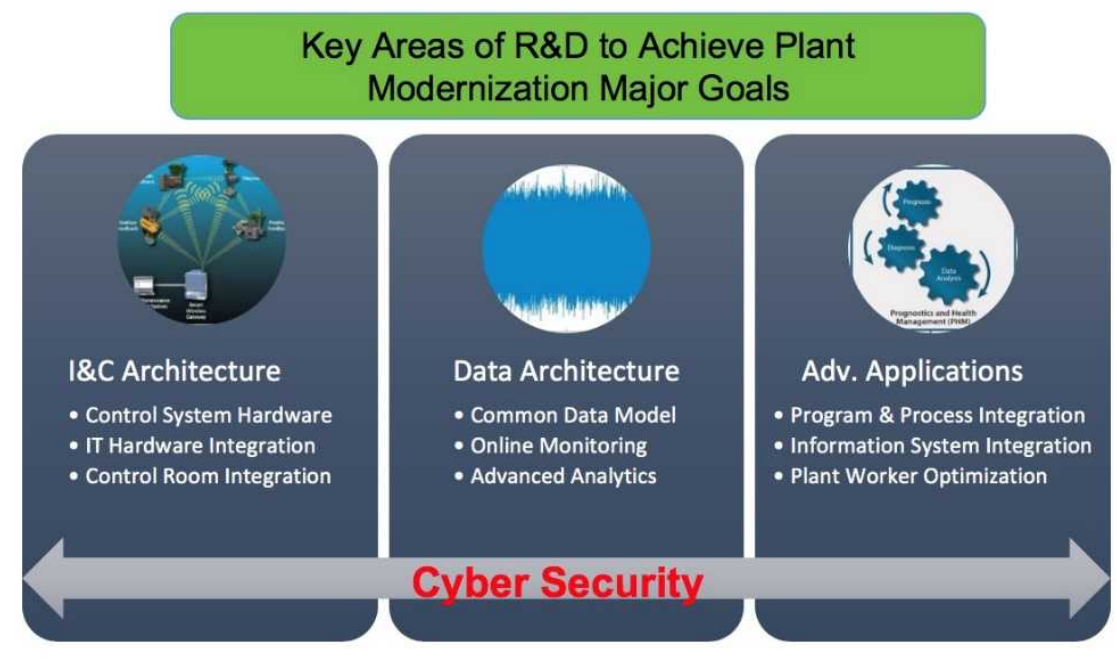

Figure 1. Key R\&D areas to achieve plant modernization.

Efforts are underway in the LWRS Program to develop and introduce a number of transformative technologies to enable plant modernization, to facilitate more-efficient work in our plants, even to diversify the products from the energy produced from nuclear power plants. The LWRS program aims to enable plants to impact the revenue/cost gap by introducing technologies and operational efficiencies that transform the organization and the methods for nuclear power plant energy production. To this end, the Nuclear Innovation Workshop described here served as a pivotal effort to initiate the development of an 
effective and impactful platform for sharing ideas and lessons learned in innovation space across the nuclear industry.

\subsection{Workshop Objectives}

The objectives of this workshop were to:

- Promote an innovation ecosystem that reduces cost and risk through early evaluations, strategic monitoring of available technologies across industries, and implementation of the processes, teams, and organizational capabilities necessary for successful discovery and development of innovative plant technologies

- Provide hands-on activities that demonstrate the value of using advanced tools, methods, and capabilities, such as those offered by the LWRS Program, to overcome challenges in innovation

- Provide a platform to enable broad innovation in the nuclear industry by capturing industry-wide experience from utilities', innovation partners', and research organizations' perspectives

- Enable industry leaders the opportunity for networking and sharing of their experiences about the challenges, solutions, and best practices in innovation

- Take inspiration from other industries to push the envelope for transformation in the nuclear industry.

These objectives were supported through facilitated discussions and industry presentations within the context of a four-phased approach to nuclear innovation described next. Appendix A provides details of the workshop's agenda. Section 1.2 provides background about the unique characteristics of the nuclear industry and how these characteristics shape the ways to which innovation should be described, implemented, and sustained. This content was presented at the workshop to support the facilitated discussions.

\subsection{Innovation in the Nuclear Industry}

The concept of innovation is sometimes portrayed as some idea, developed on a whim and perhaps drawn up on a napkin sketch, that radically changes how something is done. While it is possible for an innovative idea to develop in this way, most innovation initiatives are successful through the establishment of a systematic process that is goal driven. For businesses, an important goal is often to drive change to better adapt to an ever-evolving landscape, influenced by new competition (e.g., lower costs), changing demands, social changes and pressure, and availability of new technologies.

Within the nuclear industry, a significant challenge entails maintaining the economic viability of key plant functional areas, such as operations, maintenance, and support. For instance, most existing operating reactors have sought and received approval for their first license extensions from the U.S. Nuclear Regulatory Commission (NRC), indicating long-term interest and commitment for continued operation. However, the conditions surrounding a second license term has created markedly different circumstances from the first. These include market demand and prices that remain flat in many parts of the U.S., coupled with economic competition from other sources of energy such as renewables and unconventional production methods for gas. The traditional sociotechnical infrastructure of the existing U.S. nuclear fleet that once required large workforces and was part of a regulated market now greatly challenges the economical sustainability of these plants. Indeed, the nuclear industry recognizes the need to innovate across key functional areas, but is challenged in effectively identifying, selecting, implementing, and sustaining meaningful change. Thus, a vital question that needs to be considered across the nuclear industry entails how to effectively manage necessary innovation to improve the economic viability of the existing U.S. nuclear fleet.

The culture of an industry or organization plays a significant role in influencing how innovation is managed and executed. That is, the degree to which innovation is accepted to be implemented 
incrementally (i.e., first-order change) versus radically (i.e., second-order change) depends on the risks (or perceived risks) and attitudes that are encompassed in changing previously established processes, programs, and technologies. In the nuclear industry, notable characteristics that challenge radical change include the strong nuclear-safety culture, a high degree of regulation, and a culture adverse to change due to associated risks. Any change to a nuclear power plant, whether it be technology- or process-focused, must be evaluated and judged not to affect safety adversely. Changes must also be evaluated to confirm regulatory compliance, and they must be introduced in a way that will overcome the strong tendency of the nuclear organization to avoid any perceived risk. Innovation leaders within the nuclear industry are thus faced with two opposing forces: [1] the critical need to change and [2] strong organizational resistance to change.

To address these opposing forces faced by the nuclear industry, transforming business needs into change requires both a top-down and bottom-up approach. Hence, innovation leaders must engage their organizations and become change champions who engage and enable to entire organization to implement and sustain transformation. The implementation of both top-down and bottom-up approaches are captured in Kotter's eight-step change model, illustrated in Figure 2.

\section{Kotter's 8-Step Change Model for Transformation Change}

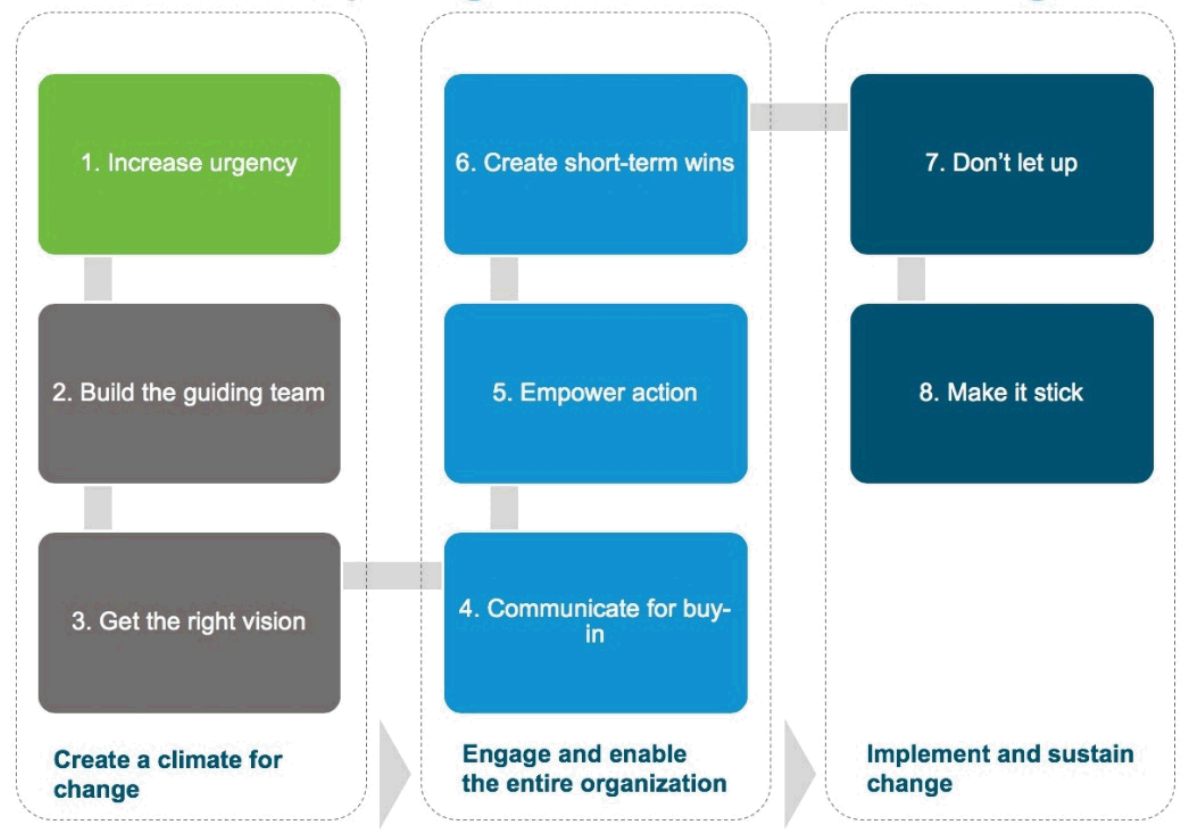

Figure 2. Kotter's eight-step model for transformational change (Kotter 2007).

Kotter's model comprises eight steps that are captured in three overarching phases. The first phase pertains to creating a climate for change; this phase is largely a top-down process that requires leaders in innovation to champion the efforts of promoting change across the organization. Step 1 regards increasing the sense of urgency for change and entails examining the market and competitive realities, as well as identifying and discussing crises, potential crises, and significant opportunities for improvement. Put simply, Step 1 requires change champions to communicate throughout the organization that the traditional business-as-usual mindset is no longer acceptable in sustaining economic viability. Step 2 specifies that a guiding group be built that has enough power to lead change across an organization and works together as a team. Kotter suggests that whenever some critical mass is not achieved early on, there will not be enough influence to support change. Step 3, "getting the vision right," entails successfully communicating a clear and compelling statement of where change leads. This message should help direct the change effort and include strategies for achieving the stated vision. 
The second phase regards engaging the entire organization for change. This phase supports bottom-up decision-making, through which each member of the organization works towards implementing and sustaining change using a common vision. Communicating the vision (Step 4) is critical for the organization to gain buy-in to change. Communication should be given on a continuous basis, clearly describing the vision and strategy for change. Next, empowering action for change (Step 5) is necessary to remove barriers that may impede momentum to innovation. For example, ambiguous and opposing requirements that obstruct innovation should be removed to empower goal-driven action that supports change. Some calculated risk should be adopted by the organization to empower an action for change. Further, innovation should be systematically implemented and bolstered through the creation of shortterm wins (Step 6). Transformation takes time and often occurs iteratively. Setting short-term goals that encompass an overarching vision provides critical feedback to the people engaging in change; this feedback is necessary for motivation and a continued sense of urgency. Finally, it is important that efforts towards change are not shortsighted (Step 7). Creating the climate for change (Steps 1-3) is most vital in creating the momentum needed for continued change. Misalignment across an organization due to a lack of urgency, an underdeveloped team, or an unclear vision can negatively affect the continued strategy to innovate. Initiatives developed for change should be anchored in the organization's culture (Step 8); change sticks when it becomes the new business as usual.

Another important consideration pertains to the areas of innovation add greatest value. In this sense, innovation can be described as change to products, processes, and programs (Figure 3).

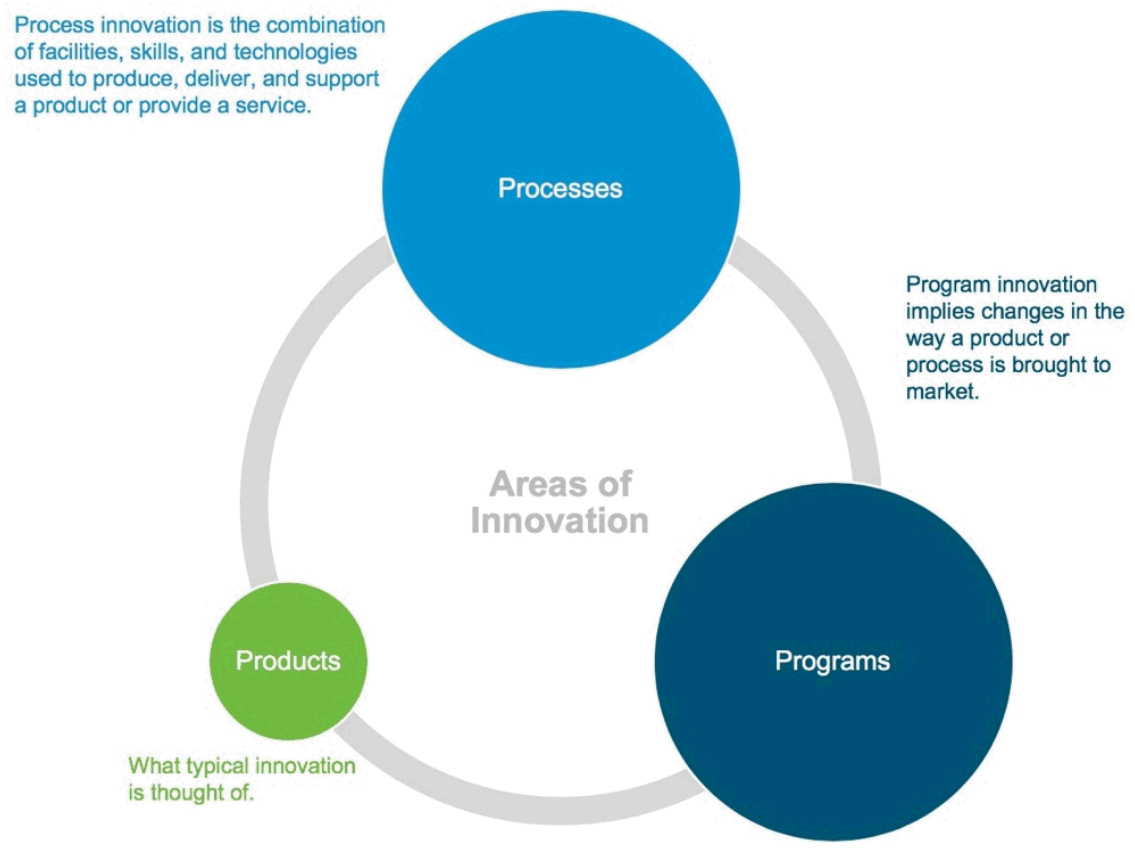

Figure 3. Three types of innovation.

Product transformation is what is traditionally thought of as innovation; it entails the development of a new product or feature to an existing product. Process innovation is the combination of facilities, skills, and technologies used to produce, deliver, and support a product or provide a service. The invention of the assembly line is an example of process innovation. Finally, program innovation is the change in a way a product or process is brought to the market. The focus of program innovation thus aims to change the culture of an organization so as to implement and sustain change.

Figure 3 illustrates these three types of innovation as they affect the nuclear industry. The size of each node illustrates the extent to which focus is needed for successful transformation. Business needs should drive nuclear innovation. A sense of urgency for change is critical for the economic viability of the U.S. 
nuclear fleet. Change ought not adversely impact safety, must be implemented with minimal risk, and needs to reduce operational costs; these factors are absolutely necessary for the existing fleet to remain cost-effective compared to other sources of energy. Product innovation is of less importance because much of the existing landscape of available technologies can be leveraged and repurposed for solving the unique challenges of the nuclear industry. By leveraging reliable off-the-shelf products, the nuclear industry can reduce the overall risks inherent to the $\mathrm{R} \& \mathrm{D}$ required to commercialize prospective technologies. Process and program innovation are most valuable for nuclear innovation because these types of innovation are purposefully driven by business needs and have wide impact on the technological, organizational, and process aspects of key functional areas. However, careful understanding of the purpose, requirements, and previous challenges in innovation for each functional area is vital for successful and sustainable transformation. Moreover, innovation leaders must evaluate technology and organizational capabilities to develop innovation needs and gaps.

To support the facilitation of evaluating technology and organizational capabilities, a process (i.e., herein described as the conduct of nuclear innovation) was presented at the workshop; it covers four iterative phases (Figure 4). Each phase is described in the following subsections.

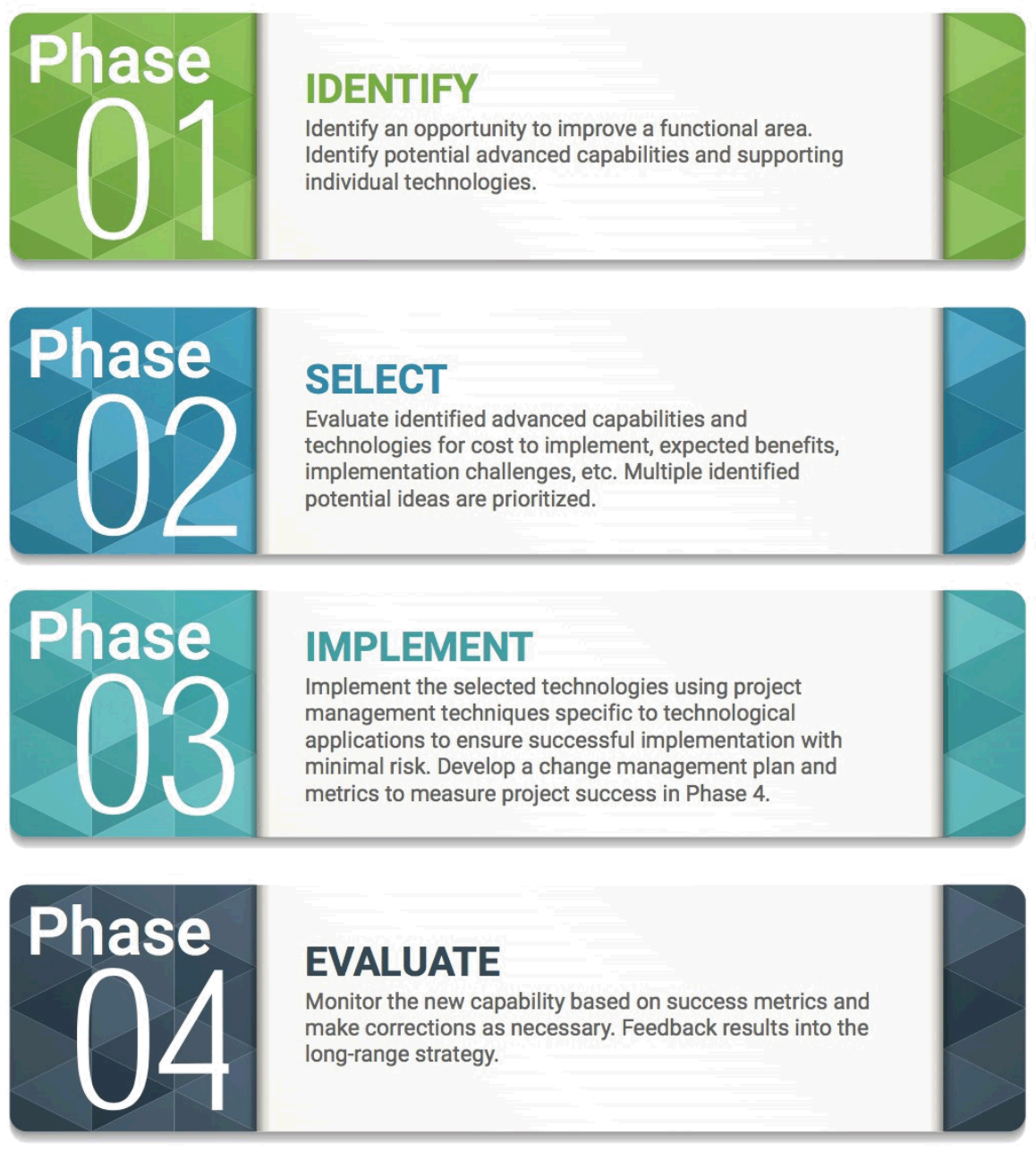

Figure 4. Four-phased framework for nuclear innovation.

\subsubsection{Phase 1: Identify}

The first phase focuses on identifying an opportunity to improve a functional area. Potential advanced capabilities and integrated technologies and applicable base technologies are also identified as part of 
improving the functional area. Figure 5 illustrates a roadmap of these different layers as they each fit within the business-driven nuclear-innovation framework. One intent of the roadmap is to eventually serve as a web-based decision-support tool that will help identify applicable advanced capabilities and technologies that support a specific functional area. The roadmap will serve as a dashboard where each label will provide clickable content. Upon selecting a particular page, detailed information will be provided. The information provided will be specific to a functional area, an advanced capability or integrated technology, or a base technology. Examples of the content supporting advanced capabilities and integrated technologies can be found in Appendix $\mathrm{C}$ whereas examples of content for base technologies can be found in Appendix D. The example content from the pages in Appendixes C and D are intended to eventually populate landing pages for this web-based tool.

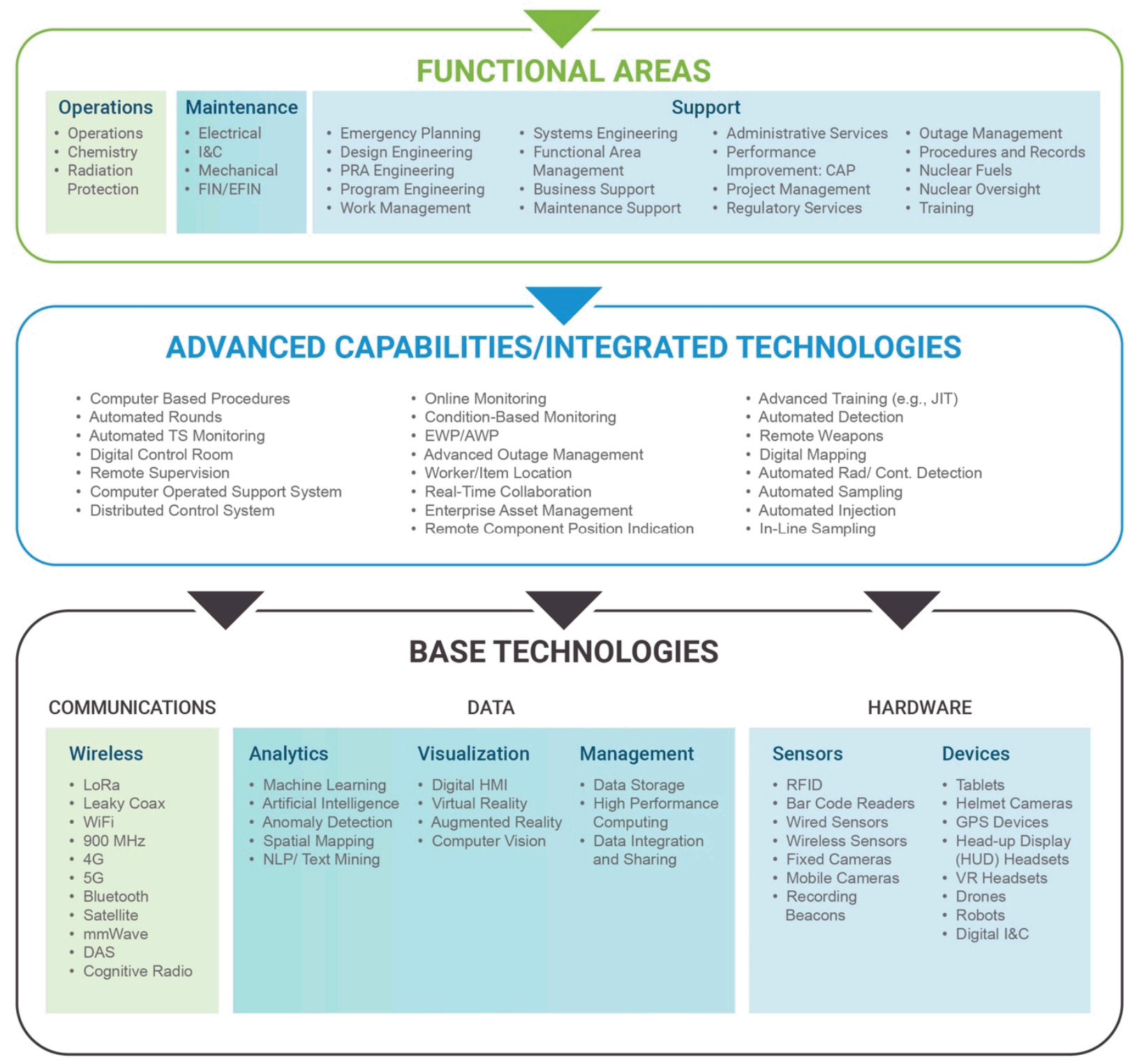

Figure 5. Roadmap for nuclear innovation.

One way the roadmap will help identify opportunities for improving a given functional area is through the interlinking layers of the roadmap, which will allow users to understand how certain 
technologies can support advanced capabilities to improve a functional area. In this manner, the tool will promote strategic technology identification and selection based how each technology supports an advanced capability that improves a business-driven functional area. Figure 6 illustrates an example architecture of these three layers under the Operations functional area. Each colored flow stream represents a specific mapping across the three layers of the roadmap. This type of mapping is a design that will be reflected in the web portal's dashboard through various cues, such as highlighting the related information upon hovering and lists of interrelated technologies, capabilities, and functional areas on each landing page.

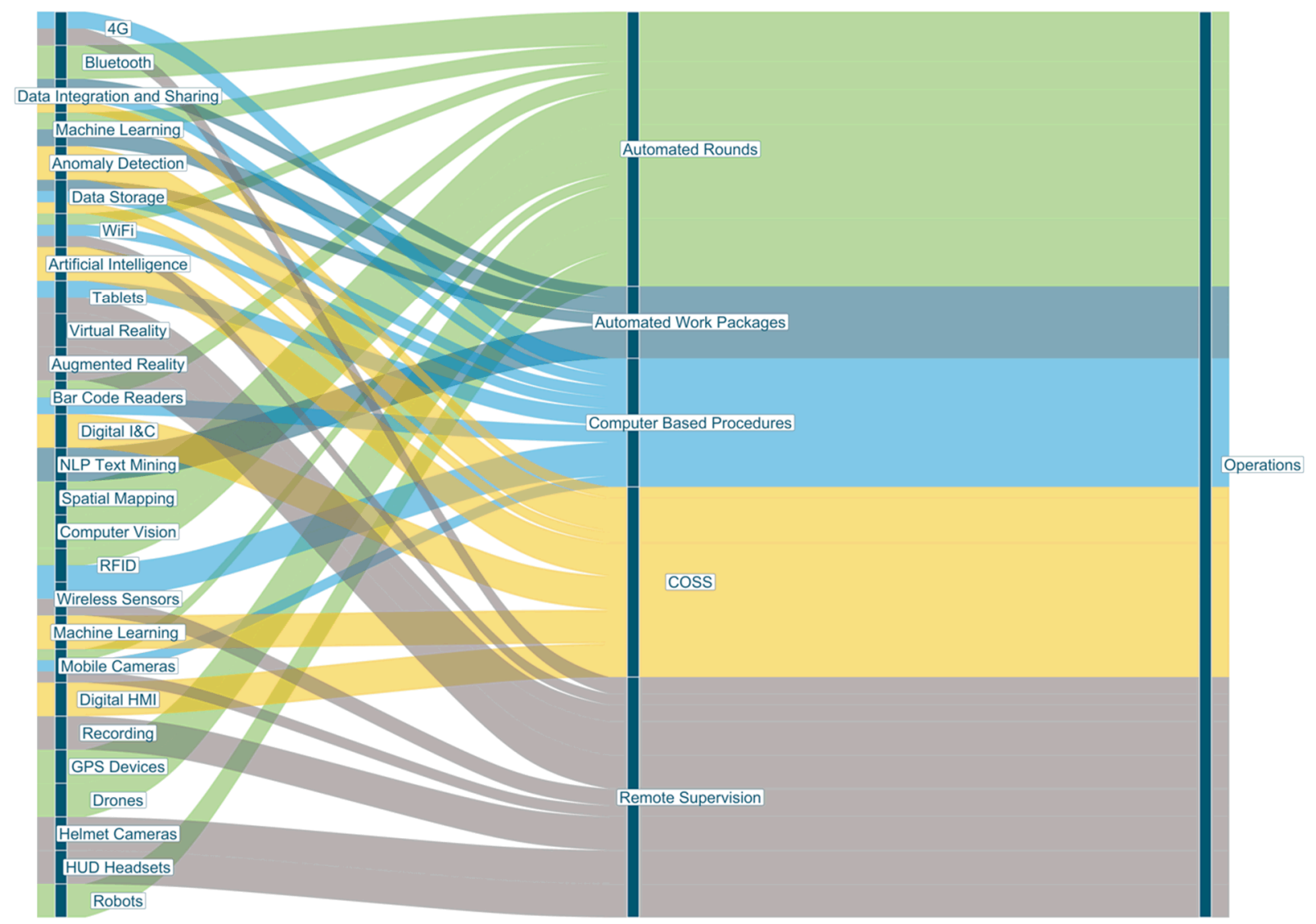

Figure 6. Example interrelations of the base technologies, advanced capabilities, and functional areas.

Additional tools that are applicable to support Phase 1 include functional-requirements analysis (FRA) and task analysis (TA). FRA can be used to identify functions that are needed to meet specific plant-safety objectives. FRA entails functional allocation (FA) where identified functions are assigned to a level of automation (automatic, shared, or manual). Successful FRA and FA account for a thorough set of inputs that are used to inform optimized allocation of automation. By identifying opportunities to improve a functional area, FRA/FA finds optimal allocations of functions that maximize the strengths of automation and human capabilities. Additionally, FRA/FA identifies important human actions that can be further examined through TA. TA is a broad category of methods used to systematically document human interactions with an existing or proposed system. TA can be used to inform the identification and selection of new technologies or processes that enhance human-system interaction. A key outcome of TA in innovation is the systematic descriptions of actions end users must undertake to accomplish a task; this information is used as further input in developing new design ideas that further support these task 
requirements. Different processes or possible technologies that are being considered that support an advanced capability can thus be formally documented regarding how they will be interfaced by the end users. This information serves as a technical basis for certain design decisions that ensure the users' physical and cognitive capabilities are considered. Appendix E provides further details of the benefits of FRA/FA and TA in nuclear innovation.

\subsubsection{Phase 2: Select}

Phase 2 includes evaluating the identified advanced capabilities and base technologies for their cost to implement, expected benefits, challenges to implement, and other key considerations that support selection. The key outcome of Phase 2 entails prioritization of multiple identified ideas based on their cost, value, and risk to implement. Possible tools that can be used to support Phase 2 include requirements gathering, cost-benefit analysis, risk management frameworks, and selection criteria for advanced capabilities and base technologies. Important considerations for gathering requirements specific to Phase 2 are listed in Table 1.

Table 1. Important considerations for the requirements gathering process.

\begin{tabular}{|c|c|}
\hline Identify the drivers for the project & Ask: \\
\hline $\begin{array}{l}\text { Ask: } \\
\text { - "What problem are we solving?" }\end{array}$ & $\begin{array}{l}\text { - "What do we and stakeholders need and } \\
\text { want?" }\end{array}$ \\
\hline $\begin{array}{l}\text { - "How can we meet those needs while } \\
\text { causing the least amount of headache } \\
\text { and disruption to affected parties?" }\end{array}$ & $\begin{array}{l}\text { - "How can we meet those needs while } \\
\text { causing the least amount of headache } \\
\text { and disruption to affected parties?" }\end{array}$ \\
\hline $\begin{array}{l}\text { - "How can we meet those needs while } \\
\text { making everyone's job a little easier?" }\end{array}$ & $\begin{array}{l}\text { - "How can we meet those needs while } \\
\quad \text { making everyone's job a little easier?" }\end{array}$ \\
\hline $\begin{array}{l}\text { Clearly identify the goals of the project: } \\
\text { - Specify what success looks like } \\
\text { - Frame success in terms of the business } \\
\text { need } \\
\text { - Identify the people, processes, and } \\
\text { systems that connect to the new system }\end{array}$ & $\begin{array}{l}\text { - Prioritize needs based on alignment with } \\
\text { business goals } \\
\text { - Anticipate unintended consequences } \\
\text { and mitigate them }\end{array}$ \\
\hline
\end{tabular}

To summarize these points, requirements gathering helps connect inputs from the business goals to the end users' needs and desires for a solution. By creating this connection, solutions can be identified and selected that minimize conflict between these inputs. Common mistakes made in requirements gathering include misaligning the representation of certain stakeholder cohorts while entirely leaving out others. Other mistakes include coming to solutions that short-sight others' perspectives, which ultimately replace one problem with another. Finally, it is important to critically look at existing requirements put in place to understand if such are truly necessary or are in artifact of legacy processes or technologies. Through thorough requirements gathering, important information can be identified for use in other tools, such as cost-benefit analysis, risk management, and other supporting tools for selection.

\subsubsection{Phase 3: Implement}

At Phase 3, selected technologies are implemented using human-centered project-management techniques that ensure successful implementation with minimized risk. A change management plan and success metrics are also developed at this phase to measure project success in Phase 4. One theme that is emphasized for Phase 3 regards following a human-centered design (HCD) approach. HCD emphasizes implementing solutions with end users in mind, as well as considering perspectives from all involved 
disciplines (e.g., human factors, cyber, operations, etc.). To this point, HCD integrates well with other multidisciplinary approaches, like the systems-engineering process.

HCD comprises three primary sub-phases: understand, design, and evaluate (see Figure 7). For HCD to be truly effective during implementation, early and iterative involvement is highly important to enable the process to collect vital inputs that will inform future design and development cost effectively.

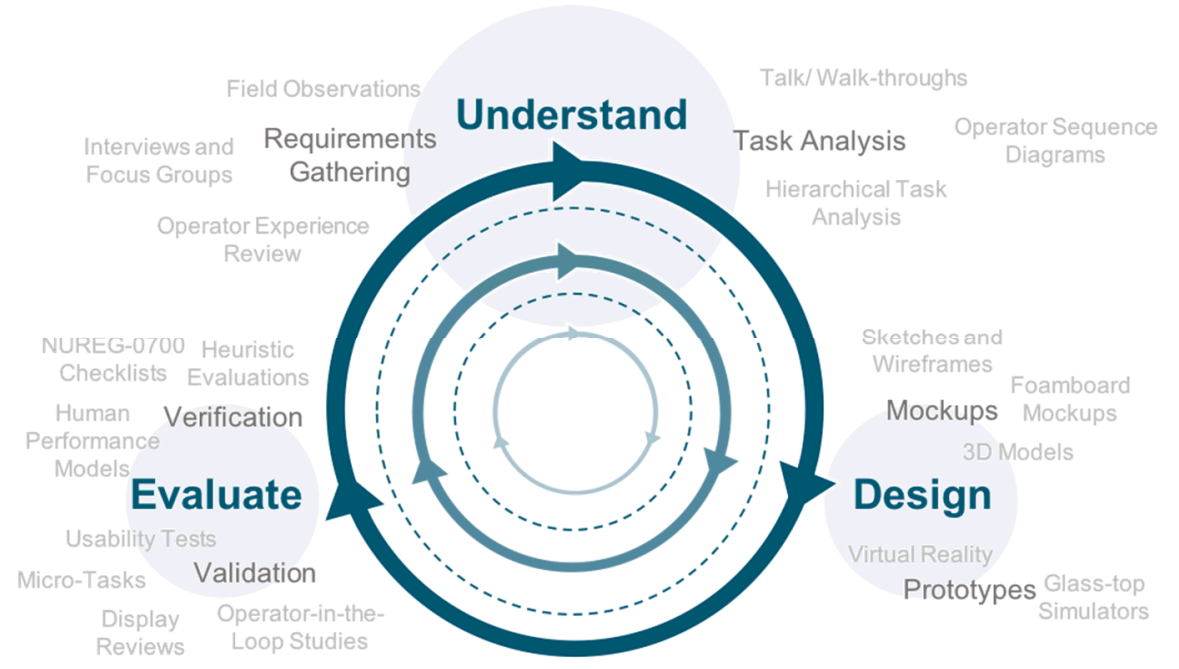

Figure 7. Human-centered design approach for nuclear innovation.

Once early inputs are collected, design activities are engaged through developing prototypes and mockups that reflect the integration of technologies within an advanced capability. Specific design questions (e.g., should this function be manual or automated?) can be answered through evaluation of the prototypes. Mockups and prototypes of varying fidelity support evaluation through both verification and validation pathways by applying various design guidelines and performing human-in-the-loop simulation.

Common mistakes in implementation include focusing on only a subset of user types, making assumptions that certain features or functions are useful or needed without vetting with end users, and failing to get early design feedback. By incorporating HCD correctly, the process can identify and mitigate critical problems earlier, enabling cost-effective implementation. Furthermore, HCD promotes buy-in to the transformation across all organizational levels (i.e., it supports the bridge between top-down and bottom-up approaches). Early design and evaluation activities provide opportunity for end users (e.g., operators of a new digital system), stakeholders, and vendors to provide their perspective and feedback for a given design in a way that enables overall human-system performance. For instance, in design workshops hosted by INL's Human System Simulation Laboratory (HSSL), key stakeholders, end users, and vendors were able to collaborate in formulating design solutions, grounded from observing or participating in operator-in-the-loop studies, that included prototypes of advanced capabilities and technologies. This testbed served as a resource for early design and evaluation of advanced capabilities to identify critical design issues before implementation, greatly reducing costs. Appendix E provides detailed information for engaging in HCD activities.

\subsubsection{Phase 4: Evaluate}

Finally, Phase 4 pertains to monitoring the newly implemented capability using success metrics. When needed, corrections can be undertaken based on tracking success metrics. Long-term strategy, focused on business needs, is developed in Phase 4 using feedback to understand a new round of identification, selection, and implementation for new capabilities and technologies. 


\section{WORKSHOP OVERVIEW}

The Nuclear Innovation Workshop was held June 26-28, 2019, at INL in Idaho Falls, Idaho. The event was hosted by the DOE-sponsored LWRS program and was attended by invitation only. This workshop aimed to help nuclear power professionals understand core qualities that are necessary for successful innovation, including the processes, teams, and organizational capabilities needed to implement advanced plant technologies. Presentations from leaders driving transformation initiatives outside of nuclear inspired participants to think beyond typical inside-the-industry ideas.

This workshop covered approaches for vetting fast-track innovation initiatives that reduce overall cost and risk. Attendees also learned how they can leverage the unique capabilities of the LWRS program to successfully overcome barriers and enhance overall benefits when developing advanced technologies. The workshop focused on lessons learned and industry perspectives from utilities, innovation partners, and research organizations related to managing plant modernization and innovation.

Key activities that supported these goals included industry presentations, facilitated discussions of each of the four innovation phases (see, e.g., Appendix F), and a post-workshop feedback survey. Furthermore, tours of INL's facilities were embedded within the workshop to demonstrate the value of the advanced tools, methods, and capabilities offered by the LWRS program. These tours included a demonstration of the capabilities of the HSSL, as well as of the Computer-Assisted Virtual Environment (CAVE). Figure 8 summarizes important activities completed at this workshop for collecting insights and demonstrations of the LWRS program's capabilities. The findings summarized in Section 3 are written to correspond with Figure 8. Refer to Appendix A for a detailed agenda of the workshop.

Day 1 (June 26, 2019)

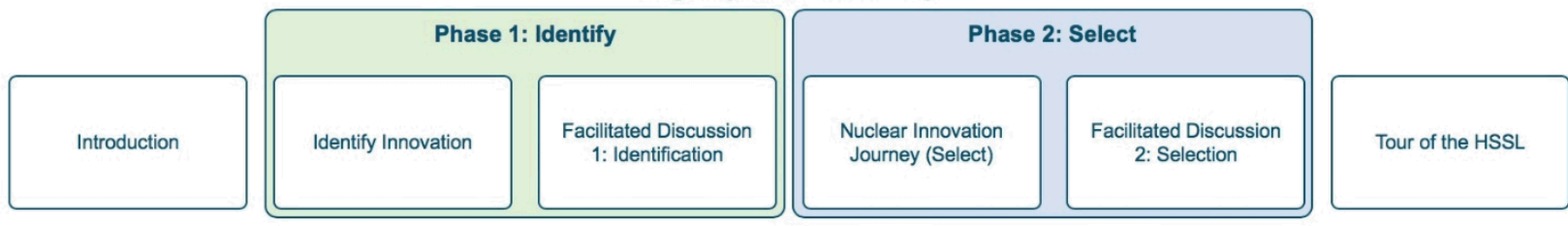

Day 2 (June 27, 2019)
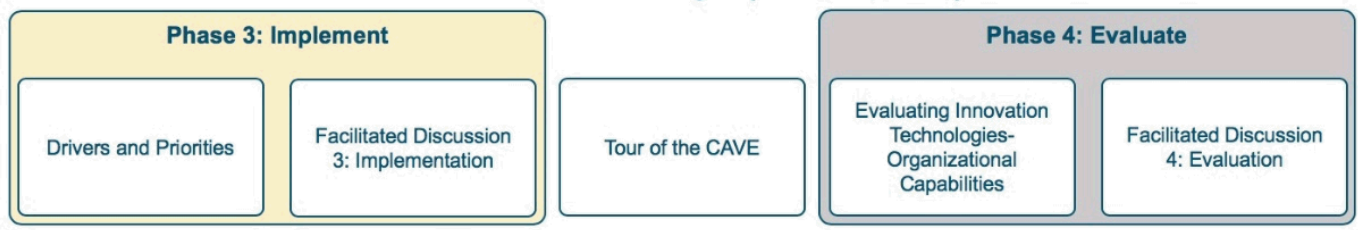

Figure 8. High-level overview of the workshop agenda. 


\section{DETAILED FINDINGS}

This section summarizes the findings collected during the Nuclear Innovation Workshop. Each subsection corresponds to findings for each workshop activity.

\subsection{Phase 1: Identify}

\subsubsection{Presentation by Dustin Greenwood (NuScale): Identify Innovation}

Dustin Greenwood (Appendix G) presented his unique experiences in the Identify phase. A notable topic that he shared regarded the need for a strategic vision-people need a reason to accept and champion change - and a process that can achieve this vision. Four key aspects of developing a successful process were discussed: [1] clarifying a good idea, [2] selecting participants, [3] ensuring engagement, and [4] taking action.

Dustin mentioned that good ideas should be bound by timing, budget, and staffing constraints so that no time is wasted on ideas that are not feasible. Supporting questions to ask include understanding who major users could be if one specific barrier were removed, what is perfection and what impedes an organization from achieving it, how would the effort be done if there were no regulations, and could a change eliminate a proposed solution's applicability. Selecting participants was also mentioned as an important aspect to the identification step. Dustin highlighted that while subject-matter experts (SMEs) are highly useful, they are only half the group; that is, some SMEs may only focus on solutions that are constrained by existing regulations without critically challenging the applicability of these regulations. An important consideration when selecting participants is, thus, to ensure a diverse set of participants are selected to provide insights from a variety of perspectives.

Ensuring engagement across brainstorming groups is important. One recommendation to ensure engaging discussion is to remove "pushy" people so as to allow others to take on leadership roles. People who are less open to others' perspectives and who dominate conversations should be grouped together. Second, the optimal size of a brainstorming group should be ten or fewer. When the group becomes too large, there is a tendency toward broken discussion (e.g., some members will not voice their opinion). Sessions should be focused on the objectives to be solved with clear ground rules for discussion (e.g., "clarity doesn't stifle innovation," as Dustin Greenwood put it). Finally, for brainstorming to be engaging, multiple sessions are important to iteratively refine ideas as needed. Greenwood suggested spending 20 to 30 minutes on a single question and keeping track of the best ideas for moving forward.

The final aspect is taking action. At this point, a narrowed list of ideas should be developed for transforming ideas to action. Feedback should focus on ideas that are supported. Communication across the organization is paramount to ensure ideas and successes are visible to everyone: "Success breeds success," said Greenwood. Having visibility of key ideas and successes is important for exploring ideas for synergy and for the success of future brainstorming sessions.

The NuScale small modular reactor (SMR) design was given as an example to highlight the application of these suggestions. Greenwood explained how NuScale leveraged the inherent simplicity of its SMR design that enhances safety through natural convection for cooling, seismic robustness, small and simplistic design, and defense-in-depth. These qualities enable reduced emergency-planning zones that allow a closer proximity to populated regions where energy is needed most. They also look beyond baseload generation. Furthermore, Greenwood highlighted how NuScale was able to rethink ways of addressing regulatory requirements, as with $50.54(\mathrm{~m})$ through novel solution of reduced operator staffing, verified and validated through integrated system validation (ISV). Greenwood closed by noting that "sometimes innovation doesn't fit in existing regulatory environment, which can create an atmosphere of 'fitting' concepts to existing regulations." 


\subsubsection{Facilitated Discussion 1: Identification}

\section{Notable Highlights}

- Identify functional areas to improve requires both a top-down (e.g., what is the business driver?) and bottom-up approach (e.g., identifying task-level opportunities for improvement).

- There are challenges with integrating top-down and bottom-up methods during Phase 1 (Identify). Tools that were identified for addressing these challenges include development of a structured database, as well as use of TA and FRA/FA to identify key tasks and functions that can be improved.

- Having an established innovation team can support fleet-wide innovation. Attendees reported that focusing on larger initiatives tends to provide greater value given effort put forth.

- Current practices for sharing innovation experience are typically done through established working groups. While this method was regarded as effective, alternative methods (e.g., a centralized resource) for exchanging ideas was suggested such as with a web-based portal.

- As utilities develop an end-state vision, there is a need for developing a roadmap that provides a framework towards reaching the end-state vision successfully with minimal risk.

The first facilitated question asked about attendees' experiences in identifying functional areas to improve.

- Representatives of Xcel Energy commented that they use both a top-down and bottom-up approach. From the top-down, costs are considered with each functional area (e.g., drive down costs $25 \%$ ). For instance, outages provide the largest point of cost savings. As for prioritizing functional areas, a topdown approach is used, including inputs such as costs, labor, and materials. Some assumptions are made in the prioritization process - for example, project staffing requirements. A database is used to support and share information for identifying and prioritizing. In parallel a bottom-up approach is completed; Xcel Energy includes SMEs iteratively in planned discussions. Using a customized spreadsheet, TA is used to identify tasks and see ways that they can be changed. In this sense, TA attempts to leverage existing activities to streamline. Xcel Energy commented that one of the challenges involves integrating cross-process aspects (e.g., across functional areas). Addressing requirements across functional areas can be an obstacle. Xcel Energy commented that it is important to approach Phase 1 from a top-down purpose-driven perspective to identify functional areas and host workshops with key people (i.e., a mixture of SMEs and others) to get multiple perspectives. Field research is also important, depending on the scope and functional area.

- Asgeir Drøivoldsmo commented that the Institute for Energy Technology (IFE) uses a similar approach. High-level roles are identified, and tasks are analyzed to determine which to keep. FRA/FA is used to allocate functions or remove work activities that do not support core functional areas. IFE uses formal methods that have been in place for 15 years in the petroleum industry.

Next, the facilitator asked, "What processes are used to fit top-down and bottom-up approaches?"

- Xcel Energy mentioned that this is one of their ultimate goals. When harvesting input from a bottomup approach, there are items that are interrelated across functional areas. To support these connections, Xcel Energy is developing a database with INL to centralize findings and deltas across processes and functional areas. 
- Paul Hippely from Westinghouse Electric Company (WEC) mentioned that most organizations' existing TAs can be leveraged; however, careful attention is needed to understand whether the level of detail provided is appropriate for identifying functional areas and related technologies (i.e., sometimes existing TAs can be too detailed). Engaging in an agile methodology did not necessarily provide noticeable benefits because scoping at this level may not be at a needed higher level. Still, FRA/FA can be used to identify areas where costs can be reduced.

- Dustin Greenwood from NuScale highlighted that a data structure is necessary for effective requirements management and associating regulations each requirement supports. Such a tool allows easy identification of parallel requirements that can be addressed.

- To this point, representatives of Xcel Energy commented that there is a significant challenge in identifying the connections between existing tasks and regulations for existing nuclear power plants. In this case, a top-down approach is helpful to target focus based on costs.

- From a vendor perspective, one challenge representatives of Curtiss-Wright mentioned was that often utilities add processes. It is important to focus on the how and why certain processes are put into place to inform decisions that influence the basis of these processes.

Next, the facilitator asked, "How has the Institute of Nuclear Power Operations (INPO) worked?"

- Dustin Greenwood commented that INPO cannot be used without analyzing requirements. A data structure is thus needed.

The facilitator then asked, "Does your organization have a formal innovation manager or group?"

- Paul Hippely noted that Exelon's Calvert Cliffs Nuclear Power Plant and corporate group has an innovation group.

- Patrick Kopfle of Dominion Energy mentioned that they have an innovation group; their group is starting with individual initiatives, but is looking to broaden. From Dominion's experience, the level of difficulty initiating a larger innovation effort is not much more than is required for smaller efforts. As a result, there is greater value in larger efforts.

- Xcel Energy commented that they have an innovation team and separate teams for individual technologies at each site.

The facilitator asked, "Where do you collect most of your operating experience (OE)? Dustin Greenwood added, "Is there an aversion to sharing OE?"

- Xcel Energy commented that OE is not competitive. It is true that, OE is built on a philosophy that facilities keep and maintain information for themselves. Sharing OE is only an afterthought; connectivity to and collaboration with $\mathrm{OE}$ is a struggle in the industry. Usually OE is shared through networking activities.

- Christopher Wiegand of the Electric Power Research Institute (EPRI) commented that he believed there was not a struggle in industry with sharing OE; rather, there is an abundance of working groups that enable sharing.

- In response, Xcel Energy agreed with the availability of working groups that enable sharing although there should be additional ways of collaborating, as is seen in the high-tech industries.

- William (Bill) Arbour of Entergy suggested that culture is one reason why the nuclear industry has not adopted alternative means of sharing OE.

- Bruce Hallbert of INL closed by suggesting that there should be a delineation between OE and experiences in innovation.

- Sean Fuller and Theresa Sutter of Curtiss-Wright mentioned that there should be lessons learned from existing initiatives (e.g., accident-tolerant fuels) that can be shared across industry. They commented 
that there also needs to be a strategic short-term and long-term (i.e., 5 and 10 year) goal in mind. Working groups can serve to provide feedback if a particular strategy is not working.

Finally, the facilitator asked if anyone from industry had developed a roadmap for innovation.

- Xcel Energy commented that they do not have an innovation roadmap yet, but do have an end-state vision based on costs. Their current approach is to learn as they work towards the end-state. They mentioned that there seem to be similar requirements across sites, which may offer an opportunity to leverage lessons learned.

- Dustin Greenwood responded that utilities should be collaborative.

- Marc Anderson of Xcel Energy agreed and commented that, "If we all do it the same way, it simplifies the process."

\subsection{Phase 2: Select}

\subsubsection{Presentation by Patrick Kopfle (Dominion): Nuclear Innovation Journey (Select)}

Patrick Kopfle of Dominion Energy (Appendix G) presented his unique experiences in the Select phase. Kopfle's presentation highlighted the importance of understanding maturity when selecting a technology as this has impact on subsequent R\&D needed and final costs. Further, Kopfle explained three importance components for selection. These included [1] developing a strong business case, [2] identifying a strong champion for innovation, and [3] identifying strong drivers to carry forward innovation.

Kopfle noted that the top reasons why startups and innovations in nuclear fail are that no market need exists or not enough funding was available for subsequent support. Patrick mentioned that selection should be strategic, organized, have a clear roadmap, consider regulatory and other barriers, and have a strong business case with definitive return on investment (ROI). He said that a common mistake is "chasing the shiny object," or selecting technology impetuously without carefully understanding the business case: what value the inclusion of such technology might provide. An example is the selection of drones, a popular technology, for performing certain tasks that could have been supported in a more costeffective way. Patrick advised developing both a 5- and 10-year plan; the business case should also be capable of simple descriptions, like "increased revenue" or "decreased costs from labor."

Next, Kopfle mentioned the need for a strong champion. This role should be at the management or executive level to ensure the greatest influence across the organization. To this end, Patrick mentioned the need for a a strong driver that will engage the right people to move innovation forward. One tool being used by Dominion Energy to support these processes includes an 'Innovation Source' website that enables innovation team members from across the organization to provide feedback on new ideas in support of key functional areas. The tool allows users to vote and provide feedback on innovative ideas, engaging them in the selection process. Kopfle noted that this feedback, along with establishing an innovation committee, has helped ground ideas to these three components. Finally, he highlighted the importance of providing a clear roadmap for innovation. This roadmap should clearly categorize projects based on their technical maturity (e.g., mature, emerging, or R\&D). 


\subsubsection{Facilitated Discussion 2: Select}

\section{Notable Highlights}

- One difference between new builds and existing plants when developing a long-term plan for data structure includes integrating new data with the existing infrastructure (for existing plants). For existing plants, this integration has been a challenge.

- One major industry challenge in anticipating obsolescence issues regards managing unique nonstandard software applications. The use of an innovation hub was mentioned as one tool that may help address this issue by promoting knowledge transfer across industry regarding lessons learned.

- Early involvement of end users is important in the selection of candidate technologies. In this process, use of testbeds like the HSSL can allow operators or other users to interact with advanced concepts early in the process, promoting greater acceptance of technology as well as further refining concepts to optimize human-system performance.

- Critically assessing the basis of existing requirements is important when selecting new capabilities to ensure innovation is not unnecessarily stifled.

- Tracking ROI is important at Phase 2. Metrics of cash flow and risk are typically considered at this stage in the process. Tools such as decision matrices have been used to support decision making to determine various technologies to support a capability.

The facilitator initiated the discussion by stating that the industry needs to think about humans first, even as technologists. If the industry does not consider humans in the selection of technologies, there will not be adequate buy-in. Further, the facilitator emphasized that a champion should not forcefully "ram [new technologies] down the throats of recipients" if the champion wishes to ensure sustained use. Rather, champions should engage recipients in a way that they can evoke their own insights and feedback into candidate solutions (i.e., see Human-Centered Approach on page 56).

Next, the facilitator talked through the hand out on how to gather requirements (see page 54) and asked Dustin Greenwood if NuScale has a long-term plan for data structure as it evolves and is used over time.

- Greenwood commented that NuScale does have a long-term plan in place. He mentioned that development differs depending on whether a new or modifications to an existing plant are planned. If the plan is for an existing plant, there is a need to integrate the data structure with the legacy structure. Dustin defined structure as the management of the many of data points (e.g., there can be one-tomany and many-to-many relationships).

Next, the facilitator asked whether anyone in industry thinks about the importance of data structures.

- Patrick Kopfle of Dominion Energy confirmed that they consider the importance of data structure. Dominion Energy engages supervisors in an industry-standard process.

The facilitator asked how industry anticipates obsolescence issues with technologies.

- Don Bosnic of Xcel Energy commented that information technology (IT) generally manages the equipment, once implemented. That is, IT determines technology-refresh rates and software-update patching. Xcel Energy is also building a hub for pushing updates, using a unified process.

- Bill Arbour of Entergy mentioned that there were challenges with the Windows 10 upgrade, such as with finding compatibility issues with unique nonstandard software (i.e., one-off applications). 
- Alison Hahn of DOE and Theresa Sutter of Curtiss-Wright both replied to the effect that both utilities and vendors are aware of these issues and are trying to anticipate for this challenge when upgrading.

The facilitator then asked what other factors are considered in a cost-benefit analysis.

- Don Bosnic mentioned total cost of ownership is a major factor to consider.

- Bill Arbour mentioned that many of the Adobe products use a subscription-based model, which was not factored in for their organization.

- Theresa Sutter of Curtiss-Wright mentioned that oftentimes utilities do not factor time to implement, including time for IT and managing the changeover.

- Craig Primer of INL highlighted that the challenges he has seen in the past regard the application space for control systems. For instance, integration of databases between legacy and new control systems has been difficult.

- Jim Hill of Xcel Energy commented that one problem on this point pertains to the shadow IT problem, where a single contractor develops a database for a specific purpose. This problem is compounded when the contractor leaves and fails to provide adequate documentation to transfer knowledge to maintain the database.

- Don Bosnic of Xcel Energy mentioned that it's very difficult to collect analytics and other information from legacy databases, especially from shadow IT systems.

- Theresa Sutter responded that an innovation hub would support addressing these challenges. She mentioned that the sharing innovation experience across different companies is critical for addressing the difficulties with integration of both legacy and new control systems.

Bruce Hallbert of INL shared his experiences with visiting various U.S. nuclear power plants. During these visits, he saw that some of these plants had done significant digital modifications. In their process, the whole plant was modified before the simulator and main control room. By the time the simulator was modified, it was a digital control room; however, the digitization of controls was merely a replication of the original control room (i.e., like-for-like replacements). These utilities would typically explain that this replication was intentional, and the operators who were involved preferred minimal change. Further, the regulator did not have a problem with the upgrade, so it offered the path of least resistance.

To this end, it is worth noting the Alison Hahn emphasized that one methodology that can be used to promote greater technology acceptance for advanced concepts is through early evaluations using test beds like the LWRS program's HSSL, which is a full-scope and fully configural simulator testbed. Jeffrey Joe of INL further shared experiences with INL's collaboration with Dominion Energy's early involvement with operators. Dominion provided their operators opportunity to demo advanced capabilities from the HSSL, as well as from other vendors including WEC and Mitsubishi. By giving operators this early exposure, Dominion experienced operators' championing advanced capabilities that, despite requiring greater familiarization and training, will benefit the plant long term.

Next, the facilitator asked if utilities follow a formal process when writing requirements.

- Don Bosnic commented that Xcel Energy has a formal process by which requirements are reviewed line-by-line. When there is a change request, oftentimes there is a barrier at some level.

- The facilitator mentioned that an important aspect to consider when referring to existing requirements is determining why a requirement exists in a certain form. Examples given include minimal staffing requirements, as well as circle-slash requirements for computed-based procedures.

The facilitator then asked how ROI is captured, including use of metrics. 
- Don Bosnic commented that utilities are getting savvy in developing cash-flow analysis. As they do, balancing short- and long-term gains is important.

- Bill Arbour commented that risk, among cost, is a major contributor for ROI. With risk, however, metrics typically only look at risks of selecting and implementing a new capability rather than also looking at risks of not selecting and implementing it.

- Christopher Wiegand of EPRI commented the net present value (NPV) is captured while evaluating risk. He commented that Duke Energy used NPV with their transformers. Christopher Wiegand highlighted that while NPV is typically employed for equipment, NPV could also be used for innovation (though this is more difficult).

Finally, the facilitator asked if a threshold is used to determining costs for selecting capabilities.

- Don Bosnic mentioned that Xcel Energy uses a decision matrix to help with the analysis. He commented that the tool helps with focusing on important considerations.

\subsection{Phase 3: Implement}

\subsubsection{Presentation by James Hill (Xcel Energy): Drivers and Priorities}

Jim Hill of Xcel Energy (Appendix G) presented his experiences in Phase 3, particularly with focus on the requirements for successfully integrating plant operations and IT. His presentation began by highlighting some of the key disparities between operations and IT regarding their fundamental philosophies. That is, plant operations focuses on maintenance, operations, and engineering, where objectives are met tactically while operators remain open to innovation and problem solving. Conversely, IT functions strategically and favors consistency and standardization for control and stability of infrastructure and services. Implementation of any advanced capability that impacts both plant operations and IT must reconcile these cultural differences.

Hill provided several solutions. From a plant operations standpoint, support for managing this perspectives gap includes engaging IT early in project planning activities through senior management, participating in strategic planning and technology selection, developing and maintaining situational awareness of various IT projects and their strategic vision, driving IT to innovate to reduce costs, and establishing champions of technology who are senior management (e.g., vice presidents and chief Nuclear officers). For IT, potential solutions include involving operations in strategic planning, considering the drivers for plant operations, balancing standardization with innovation, and establishing innovation cycles that include input from plant operations.

Hill then pointed out that one challenge with bridging plant operations and IT pertains to their differences in accountabilities. That is, plant operations, as an organization, is influenced by site vice presidents and other related roles. Plant operations is accountable for operational efficiencies, reliability, and safety of the plant. On the other hand, IT is influenced primary by the chief executive officer and the chief information officer. The primary accountabilities of IT include technology standardization and service-level commitments. Hill also provided recommended solutions to address this challenge, both from a plant operations and IT viewpoint. For both organizations, it is important to seek common accountabilities. Common accountabilities should be driven to the lowest level. Plant operations should avoid positioning IT as a service; likewise, IT should avoid positioning plant operations as a customer. Hill then pointed out that, for both groups, day-to-day operations should be segregated to help bridge the organizations.

Hill discussed the overarching goals of Xcel Energy that drive innovation, including leading the clean-energy transition through increasing renewable and carbon-free energy to $61 \%$ by 2020 and $80 \%$ by 2030. Xcel Energy needs nuclear energy to be successful in order to meet these goals. A key performance indicator (KPI) entails reducing costs per megawatt (MW) to ensure cost-effectiveness. A key driver for reducing costs per MW entails reducing total costs for operations and maintenance (O\&M). Hence, Xcel 
Energy's business strategy for innovation across their nuclear-energy portfolio is focused on reducing O\&M costs, which will consequently reduce costs per MW that supports the mission of leading the cleanenergy transition.

Hill highlighted that innovation can be collectively described as making a meaningful change to improve services, processes, or organizational effectiveness and create new value for stakeholders. For Xcel Energy, meaningful change means supporting their overall nuclear business strategy and understanding how the change will reduce costs. Hill discussed that reducing total O\&M costs entails reducing required staff size for operating and maintaining the plant. One primary solution to support staff reduction entails successful implementation of an electronic work package (eWP). With proper implementation, eWP can reduce costs through automating manual entries, linking databases, and eliminating cumulative-impact activities. Hill emphasized that, in order for the successful implementation of the eWP, early involvement of IT is necessary. In the initial attempt for implementing eWP at Xcel Energy, this did not happen. Consequently, the end result was that the existing format was merely presented as a Portable Document Format (PDF) file on a tablet. This end result unfavorably demanded substantial rework, requiring Xcel Energy to revisit the Identify and Select phases.

Jim Hill mentioned that one result from Xcel Energy's initial implementation of the eWP was the establishment of an innovation engineering team by Vice President and Chief Nuclear Officer Tim O'Connor. In this effort, one of the primary goals was to seek a process to successfully integrate IT in the implementation process. As a result, Xcel Energy formed One Xcel Energy Way (XE1), a continuous improvement process. XE1 supports idea input, as well as business assessment and value determination of prospective ideas. The entire purpose of XE1 is to align innovation initiatives to business strategy.

Hill emphasized the importance of a focused strategy, driven by business concerns, at the implementation phase. Implementation of a standardized design processes such as IP-ENG-001 (Standard Design Chance), IP-ENG-004 (Standard Digital Engineering Process), and EPRI's Digital Engineering Guide (DEG) described in EPRI 3002011816 are integral resources to support a focused strategy. Hill also mentioned the importance of process management to establish performance excellence. Excellence is not achieved from rote benchmarking of other organizations. Rather, process management values process ownership and improvement within the organization's culture. Merely adopting another organization's culture without understanding why that culture was successful in continuous improvement will not guarantee effectiveness. To this end, a technology-centered solution will not be effective if it just attempts to automate misunderstood and mismanaged processes.

Successful implementation requires systems thinking, in which the collection of processes is thought of as a system. In identifying, selecting, and implementing a technology, systems thinking emphasizes answering what needs to be supported, who are the users, and how is the proposed solution helping the users. Effective teams that support systems thinking are cross-functional. These teams cut across multiple functional areas when developing innovation solutions. 


\subsubsection{Facilitated Discussion 3: Implementation}

\section{Notable Highlights}

- Successful implementation requires systems thinking: key stakeholders are identified and considered for important input. Cross-functional teams are established that consider input across multiple functional areas. Further, users of the innovative solution are involved early in the development and implementation process.

- Establishing and maintaining early coordination between engineering, operations, and IT is important for successful implementation of a technology or process.

- Early understanding of user needs is critical in implementation. This effort goes beyond merely collecting their preferences. Having a systematic and science-based approach that balances human-system performance is important in leveraging the capabilities of new technologies and processes that ensure business needs are met. To this end, following a systems engineering approach is integral in ensuring all technical considerations are addressed.

- An agile methodology may support implementation by enabling an iterative designfeedback loop that accounts for input from various engineering disciplines, key stakeholders, and the end users. Including end users in iterative prototyping cycles can help key users understand how best to take advantage of the new capability.

- Having a change-management plan in place can provide a structured way of coordinating efforts across involvement organizations.

- Creating a shared desire for change across an organization and understanding of the new capability is important for managing expectations with a new capability.

The facilitator initiated the discussion of the implementation phase by providing attendees with printed information sheets about tools that can be used at Phase 3 to support an overarching HCD process (refer Figure 7). These tools are provided on pages 56-58 of Appendix E. The facilitator emphasized how the HCD process aligns with Jim Hill's message through its emphasis on collecting input from key stakeholders, developing a cross-functional team, and involving the actual users affected by the innovation.

Lead of the Plant Modernization LWRS Pathway Craig Primer then posed a question, "Are innovation projects managed differently than capital projects?"

- Patrick Kopfle of Dominion Energy indicated larger innovation projects are managed as large capital projects. These projects also have management sponsors of implementation. Innovation groups work to get the project approved and then follow through implementation through close coordination with IT.

Shawn St. Germain of INL asked if, through INL's non-disclosure agreement (NDA), INL could have access to Xcel Energy's long-term roadmap.

- Xcel Energy representatives indicated that they should be able to share their roadmap.

Craig Primer asked how the Nuclear Information Technology Strategic Leadership (NITSL) supports coordination between engineering, operations, and IT.

- Jim Hill pointed out NITSL alignment on expanding Wi-Fi as a network technology. It is more universal than other network technologies such as radio-frequency identification (RFID), $900 \mathrm{MHz}$, 4G, or 5G. Hill mentioned that Southern Energy has paused implementation of RFID.

The facilitator then asked how end-user experience is being evaluated during implementation and whether there were any particular challenges. 
- Representatives from Xcel Energy commented that one of the shortcomings from their initial implementation of the eWP was the lack of user involvement early on, which cost them in the long run. For instance, they mentioned that Xcel Energy initially treated their scope as a one-size-fits-all solution and didn't understand the needs of the broader user base, which were tied to their several requirements. A key contributor to this misalignment was failure to recognize the need to change the overall culture to foster innovation. One proposed methodology to solve this problem was an agile process, and another was a continuous-improvement process to rapidly evaluate ideas and foster innovation.

Katya Le Blanc of INL asked if any of the utilities or vendors had formal expertise in human factors engineering to support requirements gathering and early design and evaluation involvement for implementation of innovation initiatives.

- There was a consensus among both utility and vendor groups that an absence of expertise in human factors resources internally characterized their efforts. Xcel Energy mentioned that lacking this degree of expertise while pursuing large projects in innovation space introduces additional project risks and self-imposes process restrictions that limit overall effectiveness of the project.

- Xcel Energy commented that collecting data from end users is important, but not the only consideration. Having a science-based selection of technologies is preferable because initial feedback from users may not be representative of feelings about the final solution after refinement.

- Craig Primer mentioned that an agile process can benefit the process of collecting end-user feedback and ensuring multiple disciplines are aligned in an implementation strategy.

- Patrick Kopfle mentioned that they are not using agile process. He then asked Xcel Energy if they are using agile for innovation. Xcel Energy indicated that they are not using agile yet; however, Theresa Sutter of Curtiss-Wright mentioned that, as a vendor, they are receiving an increased request for supporting agile.

- Asgeir Drøivoldsmo of IFE commented that they have found value in having staff with a background in industrial design support their projects. Asgeir also mentioned that, if a new design is significantly different from the existing convention, additional prototyping with end-user involvement is necessary to help them understand how to take advantage of the new capability.

- Xcel Energy highlighted the value in having a good roll-out plan. They use a formal changemanagement process to proactively support implementation of a new capability. This point corroborated Jim Hill's message of having a focused strategy whereby all impacted organizations have a common understanding of the initiative at hand and are able to proactively plan for implementation activities, rather than merely react.

The Phase 3 discussion closed with the question of what barriers might be at play during implementation of new capabilities.

- Theresa Sutter commented that a lack of training for new products and processes is one problem they have experienced.

- Katya Le Blanc closed by mentioning that managing expectations of a new capability at implementation is important. To this end, fostering a desire for change is important to establish buy-in across all areas of the organization. This latter point ties in to the first step in Kotter's eight-step change model (Figure 2), ensuring a sense of urgency across an organization. 


\subsection{Phase 4: Evaluate}

\subsubsection{Presentation by Asgeir Drøivoldsmo (IFE): Evaluating Innovation Technologies - Organizational Capabilities}

Asgeir Drøivoldsmo of IFE (Appendix G) presented on Phase 4 by sharing his experiences in the petroleum industry. Drøivoldsmo's presentation focused on the business case for reducing overall costs through the implementation of integrated operations (IO) or real-time collaboration in operations. He pointed out that there is a \$20-25B opportunity in the Norwegian oil and gas industry to increase production. The capabilities that were considered to achieve this goal included advanced downhole equipment and smart wells, fiberoptic cable infrastructure for onshore-to-offshore, upgrading equipment that demands frequent inspection or extensive maintenance, advanced operation centers, upgrades to control and IT systems, and advanced automation functions.

The area Drøivoldsmo focused on for his presentation concerned reducing the number of required staff members offshore through integrated work processes. Drøivoldsmo illustrated the evolution of these approaches. Through a phased approach, traditional processes involving self-sustainable fields, specialized onshore units, and periodic onshore support transformed to integrated operation centers, heavily automated processes, and continuous operation. Drøivoldsmo highlighted that an important aspect of the IO transformation entailed identifying success criteria. He provided a notable illustration that summarized a framework used for defining these criteria (Figure 9).

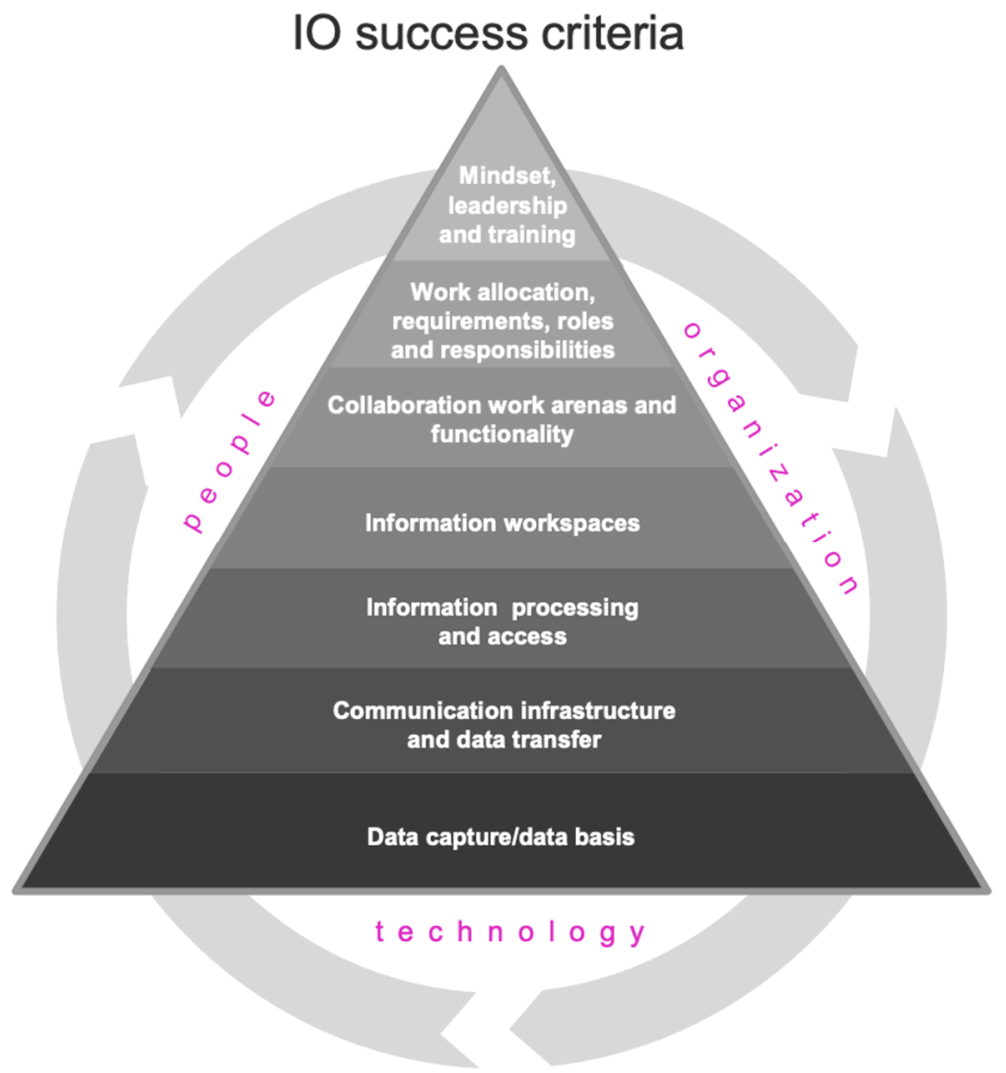

Figure 9. Identified success criteria for IO (adapted from Asgeir Drøivoldsmo’s presentation).

An important takeaway is that implementing and evaluating a new capability should go beyond focusing on the technology alone. Rather, success should be defined holistically by accounting for considerations that impact people (e.g., stakeholders and users), as well as the organization as a whole. 
Furthermore, the illustration presented by Drøivoldsmo shows a hierarchical framework for establishing success. That is, success as defined from a lower level (e.g., data capture/data basis) was necessary for the success of the subsequent level.

Drøivoldsmo highlighted important elements of ensuring success during the Evaluate phase. He emphasized that identification and development of an advanced capability needs to be grounded in meeting specific business objectives. To be identified, capabilities should provide benefit across the different silos of an organization. During development, a capability's requirements should be defined, and its maturity level should be assessed. IFE has a matrix tool to assess a capability's level of maturity; the tool comprises multiple dimensions and is based on the people capability maturity model (P-CMM). This P-CMM-based matrix allows IFE to assess a capability's maturity level across each dimension (i.e., people, processes, organization, and technology).

Drøivoldsmo shared several potential challenges of which organizations should be cognizant during Phase 4. These challenges were categorized into the four interdependent categories mentioned above: technology, people, processes, and governance. Challenges with technology pertain to issues related to having sufficient connections for transfer of data, data security, availability of equipment, functionality and maintenance issues, and compatibility of technology across different organizational boundaries. Identified challenges under people pertained to having the right competence in place to use the technology, establishing a common goal and understanding of each role, and trusting each other to ensure appropriate collaboration. Process challenges regarded a need to align or adapt the different processes involved across the organization and establish any necessary-support processes, including training and future process refinement. Governance challenges to consider include establishing contracts that address issues to support successful operations, ensuring adequate commitment to move forward, and putting the right incentives in place.

Next, Drøivoldsmo provided guidelines for running efficient and effective meetings. These guidelines support the success of the IO-transformation case study. An effective meeting should be goal oriented and held to meet a specific need in the established work process; these goals should be commonly understood by the team. Further, attendees should each have a specific role at the meeting. A fixed agenda is given for the meeting and any item mentioned outside of this defined scope is backlogged for a future meeting. Finally, meetings should be regularly evaluated to understand whether its purpose accurately reflects the overall project goal at hand.

Drøivoldsmo closed by highlighting how to reflect on a project's positive and negative results to understand future initiatives and the way the organization can learn from current results to make subsequent improvement. In his example, he listed what went well and what went wrong during the firstand second-generation work-processes integrations. Items listed were generalized beyond just technology-centered items. Rather, Drøivoldsmo focused on items that impact people, the organization, and the technology (see Figure 9). 


\subsubsection{Facilitated Discussion 4: Evaluation}

\section{Notable Highlights}

- Success should be defined up front and should holistically capture elements of the people, organization, and technology. Currently, success metrics captured in the nuclear industry tend to be higher level, focused on productivity. Example measures for ROI concern outage time and cost.

- In the nuclear industry, unanticipated successes were not typically captured (i.e., at least not consistently) using a formal process. Opportunity to develop a resource that allows utilities to share lessons learned across each phase of innovation exists and would document unanticipated successes allow their application across different innovation initiatives.

- There are socio-technical considerations with innovation in the nuclear industry. Currently in industry, there are demands for new workforce to acquire technical skills with legacy technologies, which has placed added stress to growing the talent pool.

- Lessons learned in innovation projects, such as efforts in the evaluation phase, are not formally captured or freely shared in a formal repository. Having a centralized resource to enable sharing would be valuable.

- There is opportunity to support industry in providing a clear road map for innovation to support an endpoint vision.

- Continuous improvement requires a change in culture or mindset. One approach for gaining traction in this aim is to identify smaller projects and track ideas that were adopted. Lessons learned can be pulled from these experiences.

- In the exchange of information across industry, consideration should be given to differences in objectives between utilities. Furthermore, the type of innovation and whether the change is incremental or radical should be considered when gleaning lessons learned.

The facilitator initiated the discussion by asking Asgeir Drøivoldsmo if success was formally defined from the beginning.

- Drøivoldsmo mentioned that success was defined from the beginning, based on revenue (e.g., "draining the reservoirs"). Manning to support the innovation initiative was planned as well.

- Marc Anderson and Don Bosnic of Xcel Energy tied their experiences in the nuclear industry to the conversation. They mentioned that nuclear power plants are limited by their capacity factor. Further, staff reduction has traditionally been discussed through normal attrition. Unfortunately, they pointed out that this approach is too slow and can cause reduction in staff in an unplanned way, which is not always desirable.

- Drøivoldsmo mentioned that there has been a recent push to minimize the number of disciplines needed for operations. With advances to processes and technology, one consideration has been to reduce education and training requirements.

- Paul Hippely of WEC added that another issue with staff reduction in the nuclear industry concerns whether the plant is comprised of a unionized workforce.

The facilitator transitioned by asking if anyone encountered surprise successes.

- Dustin Greenwood of NuScale responded that they do not count on surprise successes; they treat unanticipated successes as a gift. 
- Marc Anderson commented that unanticipated successes are sometimes captured at Xcel Energy in their critiques.

- Don Bosnic of Xcel Energy followed up this point with an example illustrating the converse. During outage, they found compliance with schedule and production dramatically improved by changing the number of prejob briefs and responsibilities through increasing staff members' level of ownership and involvement. This outcome was not expected; interestingly, their lessons learned were not formally captured and re-applied across different organizations. Bosnic commented that the degree of evaluation regarding the successes of a project will be influenced by its size and scope. For instance, larger initiatives, such as integrating Wi-Fi, require greater evaluation of both successes and lessons learned. Xcel is currently struggling to understand what can be achieved with Wi-Fi; more evaluation is needed.

- Patrick Kopfle of Dominion Energy added that budgets are typically adjusted upfront. In practice, successes should be measured by evaluating whether the objectives of the project were met. However, this practice is not always followed. Dominion allocates its savings, which makes it difficult to go back to evaluate.

- Drøivoldsmo mentioned that one way IFE tracks success is by measuring the amount of travel from onshore to offshore for collaboration activities.

- Don Bosnic commented that consolidating operations of multiple nuclear power plants into a single operations center would enhance overall ROI. To this end, control access would not be needed for many use cases.

The facilitator asked if travel time is captured for field operators.

- Don Bosnic responded that this level of detail is not captured and that there is no benefit collecting success metrics at this level of detail. Don mentioned that they are concerned primarily with the amount of work done.

- The facilitator then replied that in her experiences with computer-based procedures, industry's initial impressions were negative towards tracking detailed metrics such as operators' time in the field.

- Bosnic replied that there is a fundamental trust issue with tracking metrics like time in the field for the nuclear industry. By contrast, he provided the analogy of how professional sports players commonly use performance metrics to help improve their abilities. He followed up that larger global goals are what really are tracked today.

The facilitator then asked if metrics concerned with recruitment and retention might be worth considering for initiation projects.

- Bosnic commented that the industry as a whole is currently on the wrong side of the equation. He elaborated that the new workforce is required to learn older technologies such as FORTRAN. An approach for addressing this concern at Xcel Energy is through strategic staffing.

The facilitator asked if anyone had a formal process for capturing lessons learned and determining ROI at Phase 4.

- Bosnic mentioned that, for larger initiatives, there is an expectation of reduced outage duration and lower overall costs. The process is not formal, but is continuously evolving and improving through lessons learned. This process is rapidly changing without much formal documentation to support knowledge transfer. Xcel Energy is open to sharing lessons learned with industry and has shared them with a dozen utilities. He said that it is easy to differentiate between utilities interested and not interested in learning from Xcel Energy's experiences. A final point made here was that Xcel Energy currently has a clear endpoint vision, but lacks a clear roadmap to reach this vision. This was a motivation for attending the workshop. 
The facilitator followed up by asking about experiences with smaller projects.

- Jason Remer of Remer Consulting LLC followed up this question by encouraging industry to consider smaller projects: "If you're waiting for the big win, you'll never get it. Trying the smaller ones builds the culture change." Remer emphasized that continuous improvement is cumulative and is an approach that leads into transformational change. Adapting a new mindset or culture is pertinent to the success of such an approach.

- Kate Jackson of KeySource added that one metric that could determine success and track continuous change might be tracking the number of ideas that are adopted. She elaborated that this metric is usecase driven, which can help defined the scope of given technologies.

Bruce Hallbert of INL mentioned that he was impressed by the number of first movers in the industry. He commented that each approach has been slightly different, which is leading to positive outcomes with lessons learned. Halbert closed by asking if there was anything that could be done to systematically remove risk in these efforts.

- Katya Le Blanc of INL mentioned that larger projects may have tools such as agile to support innovation. However, particularly with smaller projects, one method that might help would entail building a community to exchange lessons learned.

- Patrick Kopfle added that a website would be greatly valuable. Management continuously asked him whether others in industry are involved in similar initiatives; in this case, the website would allow utilities to learn about others actively pursuing certain projects.

The facilitator concluded by asking if anyone in industry would be willing to share lessons learned from an innovation portal.

- Marc Anderson mentioned that there are different drivers for innovating in the nuclear industry, which is something to consider in an exchange of information. There could be a challenge establishing a common platform if different objectives drive the utilities.

- Dustin Greenwood suggested also considering the different types of innovation. That is, there are incremental and radical changes. Understanding what approach is being taken is an important consideration for the exchange of information.

\subsection{Wrap-up Discussion}

Craig Primer closed the workshop by providing an opportunity for those who attended to bring up any key points or concerns that were not covered during the facilitated discussions. While there were no structured questions administered at this time, the freeform discussion uncovered notable findings, including [1] insights into the interests and structure of a web-based innovation portal, [2] possible concerns to consider for subsequent innovation initiatives, and [3] next steps in developing an innovation portal and establishing an innovation group.

Primer posed the question of what sort of information should be collected for a web-based innovation portal. The information sheets provided (see Appendixes $\mathrm{C}$ and $\mathrm{D}$ ) were regarded as a good basis for content. Having contact information was suggested to allow for follow up of detailed information, as necessary. A notable comment was that the complexity of the data being requested will directly influence the web portal's ease of adding new information. A preference was expressed for less complex information on a web portal, with contact information for follow-up as needed. Another concern was the accuracy of the data being entered. That is, there would need to be a process to verify information being added to ensure validity. There was also a desire to map a utility's current state to their desired end state and have a roadmap on how to get there.

Concerns brought up at this wrap-up discussion included [1] ensuring success at the regulatory level on a given innovation initiative and [2] changing the culture to allow for shorter bursts of investment to 
innovate on smaller projects. One comment was that industry should take greater advantage of research organizations like DOE and EPRI, organizations that can serve as a resource for aligning utility initiatives to their endpoint vision. An innovation portal could support industry through providing a unified resource for the current technologies available, demonstrating how they interrelate for business-driven innovation that addresses a specific functional area. An identified advantage of a portal being hosted by the LWRS program was that this model supports a neutral platform for an honest assessment of the technologies.

Primer closed this workshop by discussing next steps in promoting an innovation ecosystem for the nuclear industry. One direction that came out of this workshop was the development of a portal to support innovation in the nuclear industry. Another direction was to establish an innovation group to routinely convene and discuss lessons learned and any innovation progress for the industry. It was understood from group discussion that current working groups like those for modernization may not have the appropriate cohort to discuss matters related to innovation. An important element that for establishing an innovation group is the inclusion of a diverse cross-section of age cohorts to ensure input that is given comprehensively reflects the needs of both the newer and more experienced workforce. A final item Primer mentioned was to further socialize the need for innovation in the nuclear industry through continual opportunities including recurrent meetings with utilities, vendors, and research organizations. These opportunities will ensure a larger sample of the nuclear community becomes aware of the mostcurrent innovation initiatives and is able give input that shapes the path forward for the nuclear industry as a whole. 


\section{SURVEY FINDINGS}

A total of fifteen ( $n=15$ ) attendees completed the feedback survey (see Appendix H). Table 2 tabulates these attendees who completed the survey by their respective organization.

Table 2. Frequency count of survey completions by organization.

\begin{tabular}{|c|c|c|c|c|c|c|c|}
\hline $\begin{array}{c}\text { Xcel } \\
\text { Energy }\end{array}$ & $\begin{array}{c}\text { Curtiss } \\
\text { Wright }\end{array}$ & $\begin{array}{c}\text { INL } \\
\text { Support }\end{array}$ & KeySource & Entergy & NuScale & $\begin{array}{c}\text { Dominion } \\
\text { Energy }\end{array}$ & $\begin{array}{c}\text { No } \\
\text { Response }\end{array}$ \\
\hline 6 & 2 & 1 & 1 & 1 & 1 & 1 & 2 \\
\hline
\end{tabular}

Findings from the survey were categorized as [1] impressions of the workshop and [2] interests in future workshop activities. These findings are reported next.

\subsection{Impressions of the Workshop}

Usefulness of the workshop was collected as a self-rating that ranged from 1 (not at all useful) to 10 (extremely useful). Overall responses were positive regarding attendee's perception of the workshop's usefulness. The mean usefulness rating was 8.14 (Figure 10), the median was 8 (i.e., indicated as a circle in Figure 10), the minimum was 7 (i.e., indicated as a white crosshair in Figure 10), and maximum was 10 (i.e., indicated as a teal crosshair in Figure 10).

\section{Overall Usefulness Rating of Workshop}

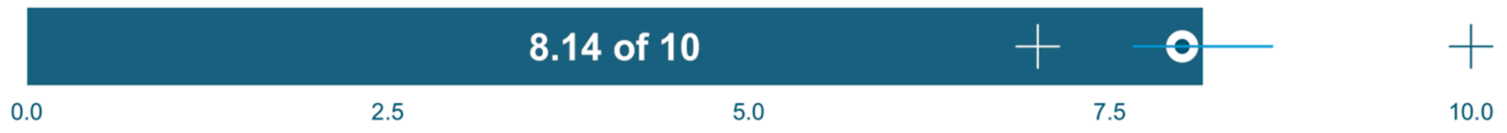

Figure 10. Average usefulness rating of workshop with $90 \%$ confidence interval.

Aspects of the workshop that attendees liked most were collected from the survey. The most frequently mentioned aspects of the workshop that attendees liked most related to the general format of the workshop; namely, attendees liked the facilitated discussions $(n=8)$ and industry presentations $(n=7)$. Notable responses were:

- "Having Dustin and Asgeir involved was great (different perspectives from basic nuclear thinking)"

- "The format was simple and having stakeholders present at the beginning of each section was engaging. The INL team did a good job bringing plant stakeholders together."

The next most commonly cited aspects that attendees liked related to the level of engagement at the workshop $(n=4)$, the framework $(n=4)$, and tours of INL's capabilities $(n=3)$. Notable comments under these themes included:

- "I thought there was a lot of engagement. I was happy to see the industry participation."

- "Framing of the meeting into 4 areas was good. Aligned with [the] speakers."

- "Exposure and understanding of INL resources and availability for further industry collaborative efforts."

Opportunities for improvement for future workshops was also collected from the survey. The most frequent comment regarded having greater utility representation $(n=6)$. A total of three utilities were represented at this initial workshop. A common rationale for desiring greater representation was to promote a more complete and broader perspective of the issues brought up during the facilitated discussions. Another comment made by attendees $(n=3)$ referred to having more specific examples used in the facilitated discussions. Attendees mentioned that having specific technologies and projects would aid the discussion. 


\subsection{Interests in Future Activities}

Most of the attendees $(n=13)$ responded that they would be interested in an innovation group should one be established (see Figure 11). One attendee responded with a response of "unsure" while another attendee did not respond to this question (i.e., this attendee did not complete the back page of the survey). The attendee who responded as unsure explained, "Depends; would need to be 'mapped' around other WG groups to prevent duplication. Would need to be focused on things that really matter (for me)."

Frequency of Those Interested in an Innovation Group

Interested

No Response

Unsure
13

Figure 11. Frequency interested in participating in an innovation group if established.

Most of the attendees $(n=11)$ responded that they were interested in an innovation portal (see Figure 12). Three attendees were unsure on the portal, and one attendee did not respond to the question. Attendees who were interested commented that having an innovation portal would be extremely helpful, especially if it promoted collaboration across industry. Notable responses for those who were unsure included:

- "For now, I would say No. A portal for the working group to engage INL in support of innovation topics, projects, and proposed funding paths would be useful."

- "If focused on topics incremental step change (innovation process risk [management])."

Frequency of Those Interested in an Innovation Portal

Interested

Unsure

No Response

\section{1}

Figure 12. Frequency interested in an innovation portal. 


\section{CONCLUSION AND NEXT STEPS}

The Nuclear Innovation Workshop set out to help nuclear power professionals understand core qualities that are necessary for successful innovation. The approach shared and discussed at this workshop considered the processes, teams, and organizational capabilities needed to implement advanced plant technologies. Key activities of the workshop included industry presentations, facilitated discussions of each of the four innovation phases (i.e., Identify, Select, Implement, and Evaluate), and a post-workshop feedback survey. Moreover, the workshop provided several tours of INL's facilities to demonstrate the value of advanced tools, methods, and capabilities offered by the LWRS program.

Key members from industry presented on their unique experiences in engaging in a given innovation phase. These presentations shared experiences not only from traditional LWR utilities, but more broadly touched on lessons learned from related sectors, such as with SMR design and from the oil and gas industry. Open conversation about key challenges and considerations for innovation in the nuclear industry was afforded through the facilitated discussions. These findings were structured around each of the four phases and are highlighted in Section 3. Further, a feedback survey was given to attendees at the close of the workshop to elicit feedback on the quality of the workshop and their impressions of future initiatives for promoting an innovation ecosystem, such as with developing a web-based innovation portal and establishing an innovation group. These findings are presented in Section 4. Finally, contact information from the attendees of this workshop, industry presentations, and all related materials can be found from the following LWRS program webpage:

https://lwrs.inl.gov/Innovation_in_the_Nuclear_Industry_June_2019/Home.aspx

Moving forward, action items that came from the workshop broadly included the development of an innovation portal, establishment of an innovation group, and continual socialization of current status and lessons learned in innovation across industry through reoccurring meetings with utilities, vendors, and research organizations. To this end, the information collected from this workshop will be used to inform these future initiatives. 


\section{REFERENCES}

Disclaimer: The references in this section comprehensively document the entirety of resources provided from the report and supporting materials of the Nuclear Innovation Workshop.

1. Boring, R. \& Lau N. (2015). Verification and Validation of Digitally Upgraded Control Rooms. (INL/EXT-15-36704). Idaho National Laboratory.

2. Electric Power Research Institute (2001). Human Performance Optimization: Emerging Management Issues and Artificial Intelligence Methods: Volume 4: Supporting Group Decision Making and Team Operations with Artificial Intelligence. EPRI, Palo Alto, CA. 1004674.

3. Electric Power Research Institute (2001). Plant Process Computer Replacement to Support Distributed Process Control: Joint STPNOC-EPRI Distributed Plant Process Computer System Project. EPRI, Palo Alto, CA. 1000804.

4. Electric Power Research Institute (2002). Implementation of On-Line Monitoring: Technical Specification Instruments. EPRI, Palo Alto, CA. 1006833.

5. Electric Power Research Institute (2002). Nuclear Maintenance Applications Center: Maintenance Work Package Training for Nuclear Utility Personnel - Student Handbook. EPRI, Palo Alto, CA. 1014533.

6. Electric Power Research Institute (2011). Guideline for On-Line Monitoring of Nuclear Power Plant Instrument Channel Performance. EPRI, Palo Alto, CA. 1022988.

7. Electric Power Research Institute (2014). Developing an Observation Program for Fossil Electric Generating Stations. EPRI, Palo Alto, CA. 3002001131.

8. Electric Power Research Institute (2014). Cost-Benefit Study for Mobile Devices for Operator Rounds and Work Order Closeout at Fossil Generating Sites. EPRI, Palo Alto, CA. 3002003898.

9. Electric Power Research Institute (2014). Pressurized Water Reactor Primary Water Chemistry Guidelines: Revision 7, Volumes 1 and 2. EPRI, Palo Alto, CA. 3002000505.

10. Electric Power Research Institute (2015). Human Factors Guidance for Control Room and Digital Human-System Interface Design and Modification: Guidelines for Planning, Specification, Design, Licensing, Implementation, Training, Operation, and Maintenance for Operating Plants and New Builds. EPRI, Palo Alto, CA. 3002004310.

11. Electric Power Research Institute (2015). Remote Monitoring Technology Guide for Radiation Protection: Field Implementation of Remote Monitoring, 2015 Revision. EPRI, Palo Alto, CA. 3002005480.

12. Electric Power Research Institute (2016). Advanced Sensing and Monitoring Options to Improve Operational Effectiveness. EPRI, Palo Alto, CA. 3002008602.

13. Electric Power Research Institute (2017). Pressurized Water Reactor Secondary Water Chemistry Guidelines-Revision 8. EPRI, Palo Alto, CA. 3002010645.

14. Electric Power Research Institute (2018). Digital Engineering Guide: Decision Making Using Systems Engineering. EPRI, Palo Alto, CA. 3002011816.

15. Gothelf, J. (2013). Lean UX: Applying lean principles to improve user experience. O'Reilly Media, Inc. 
16. Feigh, K. M., \& Pritchett, A. R. (2014). Requirements for e ective function allocation: A critical review. Journal of cognitive engineering and decision making, 8(1), 23-32.

17. Hollnagel, E., \& Bye, A. (2000). Principles for modelling function allocation. International Journal of Human-Computer Studies, 52(2), 253-265.

18. Hugo, J. \& Joe, J. (2017). Regulatory Aspects of Human Factors Engineering Process in Control Room Modernization. (INL/EXT-17-42190). Idaho National Laboratory.

19. Hugo, J., Kovesdi, C., \& Joe, J. (2018). The Strategic Value of Human Factors Engineering in Control Room Modernization. (INL/EXT-18-51365). Idaho National Laboratory.

20. IAEA (2016). Nuclear Plant Outage Optimization Strategy. (IAEA-TECDOC-1806). International Atomic Energy Agency.

21. IEEE Standard Subroutines for Computer Automated Measurement and Control (CAMAC). IEEE Std. 758-1979, 1979.

22. IEEE Standard for SCADA and Automation Systems. IEEE Std. C37.1:2007, 2007.

23. IEEE Guide for Human Factors Applications of Computerized Operating Procedure Systems (COPS) at Nuclear Power Generating Stations and Other Nuclear Facilities. IEEE Std. 17862011, 2011.

24. IEEE Guide for Computer-based Control for Hydroelectric Power Plant Automation. IEEE Std. 1249-2013, 2013.

25. IEEE Standard Ontologies for Robotics and Automation. IEEE Std. 1872-2015, 2015.

26. IEEE Standard for Robot Map Data Representation for Navigation. IEEE Std. 1873-2015, 2015.

27. ISO/IEC. "Sampling of Chemical Products for Industrial Use - Safety in Sampling," ISO/IEC 3165:1976.

28. ISO/IEC. "9241-11 Ergonomic Requirements for Office Work with Visual Display Terminals (VDT)s - Part II Guidance on Usability," ISO/IEC 9241-11,1998 (E).

29. ISO/IEC. "Industrial Automation Systems and Integration - Project Data Representation and Exchange - Part 240" ISO/IEC 10303-240:2005.

30. ISO/IEC. "Sampling of Airborne Radioactive Materials from the Stacks and Ducts of Nuclear Facilities," ISO/IEC 2889:2010.

31. Joe, J., Hanes, L., \& Kovesdi, C. (2018). Developing a Human Factors Engineering Program Plan and End State Vision to Support Full Nuclear Power Plant Modernization. (INL/EXT-18-51212). Idaho National Laboratory.

32. Kirwan, B., \& Ainsworth, L. K. (Eds.). (1992). A guide to task analysis: the task analysis working group. CRC press.

33. Kotter, J. (2007). Leading change: Why transformation efforts fail. Harvard Business Review Best of HBR 1-10.

34. Kovesdi., C., Spielman, Z., Hill, R., Le Blanc, K., \& Oxstrand, J. (2018). Development and Evaluation of the Conceptual Design for a Liquid Radiological Waste System in an Advanced Hybrid Control Room. (INL/EXT-18-51107). Idaho National Laboratory.

35. Kovesdi., C. \& Joe, J. (2016). Migration of Older to New Digital Control Systems in Nuclear Power Plant Main Control Rooms. (INL/EXT-16-38576). Idaho National Laboratory. 
36. Kovesdi, C., Joe, J., \& Boring, R. (2018). A guide for selecting appropriate human factors methods and measures in control room modernization e orts in nuclear power plants. In International Conference on Applied Human Factors and Ergonomics (pp. 441-452). Springer, Cham.

37. Le Blanc, K., Oxstrand, J. \& Joe, J. (2015). Requirements for Control Room Computer-Based Procedures for use in Hybrid Control Rooms. (INL/EXT-15-35284). Idaho National Laboratory.

38. Le Blanc, K., Kovesdi, C., Hill, R., Spielman, Z., Hansen, T., \& Oxstrand, J. (2017). Evaluation of Control Room Interface Designs to Support Modernization in Nuclear Power Plants. (INL/EXT-17-43250). Idaho National Laboratory.

39. Le Blanc, K., Hugo, J., Spielman, Z., Kovesdi, C., Hill, R., Oxstrand, J., Hansen, T., \& Rice, B. (2018). Control Room Modernization End-State Design Philosophy. (INL/EXT-18-44798). Idaho National Laboratory.

40. NEI (2000). Guidelines for 10 CFR 50.59 Evaluations. NEI 96-07 Rev 1. Washington, D.C.: Nuclear Energy Institute.

41. Oxstrand, J. \& Bly, A. (2017). Seamless Digital Environment - Data Analytics Use Case Study. (INL/EXT-17-42918). Idaho National Laboratory.

42. Pham, B., Lybeck, N., \& Agarwal, V. (2012). Online Monitoring Technical Basis and Analysis Framework for Emergency Diesel Generators - Interim Report for FY 2013. (INL/EXT-1227754). Idaho National Laboratory.

43. Rashdan, A. \& Mortenson, T. (2018). Automation Technologies Impact on the Work Process of Nuclear Power Plants. (INL/EXT-18-51457). Idaho National Laboratory.

44. Rashdan, A. \& St. Germain, S. (2018). Automation of Data Collection Methods for Online Monitoring of Nuclear Power Plants. (INL/EXT-18-51456). Idaho National Laboratory.

45. Rashdan, A., Smith, J., St. Germain, S., Ritter, C., Agarwal, V., Boring, R., Ulrich, T., Hansen, J. (2018). Development of a Technology Roadmap for Online Monitoring of Nuclear Power Plants. (INL/EXT-18-52206). Idaho National Laboratory.

46. Rashdan, A., Krome, C., St. Germain, S., Corporan, J., Ruppert, K., \& Rosenlof, J. (2019). Method and Application of Data Integration at a Nuclear Power Plant. (INL/EXT-19-54294). Idaho National Laboratory.

47. Rothwell, R. (1992). Successful industrial innovation: critical factors for the 1990s. R\&D Management, 22(3), 221-240.

48. Snyder, C. (2003). Paper prototyping: The fast and easy way to design and refine user interfaces. Morgan Kaufmann.

49. St. Germain, S., Farris, K., Whaley, A., Medema, H. \& Gertman, D. (2014). Guidelines for Implementation of an Advanced Outage Control Center to Improve Outage Coordination, Problem Resolution, and Outage Risk Management. (INL/EXT-14-33182). Idaho National Laboratory.

50. St. Germain, S. \& Hugo, J. (2016). Development of an Overview Display to Allow Advanced Outage Control Center Management to Quickly Evaluate Outage Status. (INL/EXT-16-39622). Idaho National Laboratory.

51. St. Germain, S., Hugo, J., Globbel, G., \& Reeves R. (2018). Prototype System for Detecting Interactions between Current Plant Configuration States and Component Manipulations Directed by In-Use Procedures. (INL/EXT-18-51474). Idaho National Laboratory. 
52. St. Germain, S., Hugo, J., Manic, M., \& Amarasinghe, K. (2017). Technologies for Detecting Interactions between Current Plant Configuration States and Component Manipulations Directed by In-Use Procedures. (INL/EXT-17-43234). Idaho National Laboratory.

53. Stanton, N., Salmon, P. M., \& Rafferty, L. A. (2013). Human factors methods: a practical guide for engineering and design. Ashgate Publishing, Ltd.

54. Thomas, K. \& Hallbert B. (2012). Long-Term Instrumentation, Information, and Control Systems (II\&C) Modernization Future Vision and Strategy. (INL/EXT-11-24154). Idaho National Laboratory.

55. Thomas, K., Lawrie, S., \& Niedermuller, J.M. (2016). A Business Case for Advanced Outage Management. (INL/EXT-16-38265). Idaho National Laboratory.

56. Ulrich, T., Boring, R., Phoenix, W., Dehority, E., Whiting, T., Morrell, J., \& Backstrom, R. (2012). Applying Human Factors Evaluation and Design Guidance to a Nuclear Power Plant Digital Control System. (INL/EXT-12-26787). Idaho National Laboratory.

57. Ulrich, T., Lew, R., Boring, R., Thomas, K., Rice, B., \& Poresky, C. (2017). Operator-in-theLoop Study for a Computerized Operator Support System (COSS) - Cross-System and System Independent Evaluations. (INL/EXT-17-43390). Idaho National Laboratory.

58. U.S. Nuclear Regulatory Commission. (1980). Clarification of TMI Action Plan Requirements, NUREG-0737.

59. U.S. Nuclear Regulatory Commission. (2000). Computer-Based Procedure Systems: Technical Basis and Human Factors Review Guidance, NUREG/CR-6634.

60. U.S. Nuclear Regulatory Commission. (2002). The Human Performance Evaluation Process: A Resource for Reviewing the Identification and Resolution of Human Performance Problems, NUREG/CR-6751.

61. U.S. Nuclear Regulatory Commission. (2002). Human-System Interface Design Review Guidelines, NUREG-0700, Rev. 2.

62. U.S. Nuclear Regulatory Commission. (2005). Technical Review of On-Line Monitoring Techniques for Performance Assessment, NUREG/CR-6895.

63. U.S. Nuclear Regulatory Commission. (2012). Human Factors Engineering Program Review Model, NUREG-0711, Rev. 3.

64. U.S. Nuclear Regulatory Commission. (2016). Consolidated Guidance about Materials Licenses, NUREG-1556, Vol. 1.

65. U.S. Nuclear Regulatory Commission. (2016). International Workshop on the Use of Robotic Technologies at Nuclear Facilities, NUREG/CP-0304, Vol. 1.

66. U.S. Nuclear Regulatory Commission. (2016). Standard Review Plan for the Review of Safety Analysis Reports for Nuclear Power Plants: LWR Edition-Human Factors Engineering, Chapter 18, NUREG-0800, Rev. 3. Washington, DC: U.S. Nuclear Regulatory Commission. 


\section{Appendix A}

\section{Workshop Agenda}

\section{Wednesday, June 26th, 2019}

Energy Innovation Laboratory (EIL) Meeting Center, 775 University Blvd, Idaho Falls, ID (Rooms A111-A113)

\begin{tabular}{|c|c|c|c|}
\hline Time & Subject & Location & Speaker \\
\hline $7: 30$ a.m. & $\begin{array}{l}\text { Badging - Willow Creek Building } \\
1995 \text { Fremont Avenue, Idaho Falls, ID }\end{array}$ & WCB & Casey Kovesdi, INL \\
\hline 8:00 a.m. & Welcome and Purpose of Meeting & EIL, A111-113 & $\begin{array}{l}\text { Bruce Hallbert, INL } \\
\text { Craig Primer, INL }\end{array}$ \\
\hline 8:15 a.m. & The Vision of Nuclear Innovation & EIL, A111-113 & Craig Primer \\
\hline 9:00 a.m. & $\begin{array}{l}\text { Introducing the Four Phased Approach for } \\
\text { Managing Innovation }\end{array}$ & EIL, A111-113 & Craig Primer \\
\hline 9:30 a.m. & Break (as needed) & EIL, A111-113 & \\
\hline 9:45 a.m. & Identifying Potential Technologies & EIL, A111-113 & $\begin{array}{l}\text { Dustin Greenwood, } \\
\text { NuScale }\end{array}$ \\
\hline 10:45 a.m. & Phase 1: Identification & EIL, A111-113 & $\begin{array}{l}\text { Shawn St Germain, } \\
\text { INL } \\
\text { Casey Kovesdi }\end{array}$ \\
\hline \multicolumn{4}{|c|}{$\begin{array}{l}\text { Topic: How to identify an opportunity to improve a functional area and to identify potential advanced capabilities } \\
\text { and supporting individual technologies. }\end{array}$} \\
\hline 12:15 p.m. & $\begin{array}{l}\text { Working Lunch: Overview of LWRS Research } \\
\text { Activities }\end{array}$ & EIL, A111-113 & Vaibhav Yadav, INL \\
\hline 1:15 p.m. & Selecting Candidate Technologies & EIL, A111-113 & $\begin{array}{l}\text { Patrick Kopfle, } \\
\text { Dominion }\end{array}$ \\
\hline 2:15 p.m. & Phase 2: Selection & EIL, A111-113 & $\begin{array}{l}\text { Katya Le Blanc, INL } \\
\text { Jeffrey Joe, INL }\end{array}$ \\
\hline \multicolumn{4}{|c|}{$\begin{array}{l}\text { Topic: How to evaluate identified advanced capabilities and technologies for cost to implement, expected benefits, } \\
\text { and implementation challenges. Multiple identified potential ideas are prioritized. }\end{array}$} \\
\hline 3:45 p.m. & Break & EIL, A111-113 & \\
\hline 4:00 p.m. & $\begin{array}{l}\text { Tour Human Systems Simulation Laboratory's } \\
\text { (HSSL) Capabilities in Early Evaluation of } \\
\text { Candidate Technologies }\end{array}$ & EIL, B207 & Casey Kovesdi \\
\hline 5:00 p.m. & Adjourn & & \\
\hline
\end{tabular}




\section{Thursday, June 27th, 2019}

Energy Innovation Laboratory (EIL) Meeting Center, 775 University Blvd, Idaho Falls, ID (Rooms A111-A113)

\begin{tabular}{|c|c|c|c|}
\hline Time & Subject & Location & Speaker \\
\hline 8:00 a.m. & Recap and Overview of Day 2 Agenda & EIL, A111-113 & Craig Primer \\
\hline 8:15 a.m. & Implementing Innovation Technologies & EIL, A111-113 & Jim Hill, Xcel \\
\hline 9:15 a.m. & Phase 3: Implementation & EIL, A111-113 & $\begin{array}{l}\text { Casey Kovesdi } \\
\text { Shawn St Germain }\end{array}$ \\
\hline \multicolumn{4}{|c|}{$\begin{array}{l}\text { Topic: How to implement the selected technologies using project management techniques specific to technological } \\
\text { applications to ensure successful implementation with minimal risk. How to develop a change management plan } \\
\text { and metrics to measure project success in Phase } 4 .\end{array}$} \\
\hline 10:45 a.m. & Break & EIL, A111-113 & \\
\hline 11:00 a.m. & $\begin{array}{l}\text { Tour the Computer-Assisted Virtual } \\
\text { Environment (CAVE) at CAES }\end{array}$ & CAES & Casey Kovesdi \\
\hline 12:15 p.m. & $\begin{array}{l}\text { Working Lunch: Best Practices for Validation } \\
\text { in Innovation Implementation }\end{array}$ & EIL, A111-113 & Zachary Spielman, INL \\
\hline 1:00 p.m. & Evaluating Innovation Technologies & EIL, A111-113 & $\begin{array}{l}\text { Asgeir Drøivoldsmo, } \\
\text { IFE }\end{array}$ \\
\hline 2:00 p.m. & Phase 4: Evaluation & EIL, A111-113 & $\begin{array}{l}\text { Ahmad Al Rashdan } \\
\text { Casey Kovesdi }\end{array}$ \\
\hline
\end{tabular}

Topic: How to monitor the new capability based on success metrics, make corrections as necessary, and feedback results into the long-range strategy.
3:30 p.m.
Break
EIL, A111-113
3:45 p.m.
Wrap-up: Summary Discussion of Four Phased Approach for Managing Innovation
EIL, A111-113
Katya Le Blanc
4:15 p.m.
How to Support Innovation Going Forward
EIL, A111-113
Casey Kovesdi

5:00 p.m.

Acknowledgements and Adjourn

\section{Friday, June 28th, 2019}

Energy Innovation Laboratory (EIL) Meeting Center, 775 University Blvd, Idaho Falls, ID

\begin{tabular}{llll} 
Time & \multicolumn{1}{c}{ Subject } & Location & Speaker \\
8:30 a.m. & Meet at EIL & \\
9:00 a.m. & Tour of INL's Facilities & \\
1:00 p.m. & Adjourn &
\end{tabular}




\section{Appendix B}

\section{List of Attendees}

\begin{tabular}{|c|c|c|c|c|}
\hline Name & Company & Title & Email & Phone \\
\hline Sean Fuller & Curtiss-Wright & Innovation Lead & sfuller1@curtisswright.com & $203-448-6415$ \\
\hline Theresa Sutter & Curtiss-Wright & $\begin{array}{l}\text { Director, } \\
\text { Information } \\
\text { Solutions } \\
\end{array}$ & tsutter@curtisswright.com & $727-669-3027$ \\
\hline Patrick Kopfle & Dominion Energy & $\begin{array}{l}\text { Nuclear Technology } \\
\text { \& Innovation } \\
\text { Consultant }\end{array}$ & $\begin{array}{l}\text { patrick.j.kopfle@dominione } \\
\text { nergy.com }\end{array}$ & $804-273-2794$ \\
\hline William Arbour & Entergy & Operations CFAM & warbour@entergy.com & $760-504-9071$ \\
\hline $\begin{array}{l}\text { Christopher } \\
\text { Wiegand }\end{array}$ & EPRI & $\begin{array}{l}\text { Senior Technical } \\
\text { Executive }\end{array}$ & cwiegand@epri.com & $630-659-8134$ \\
\hline Bruce Halbert & $\begin{array}{l}\text { Idaho National } \\
\text { Laboratory }\end{array}$ & Director & bruce.hallbert@inl.gov & $208-526-9867$ \\
\hline Casey Kovesdi & $\begin{array}{l}\text { Idaho National } \\
\text { Laboratory }\end{array}$ & $\begin{array}{l}\text { Human Factors } \\
\text { Scientist }\end{array}$ & Casey.Kovesdi@inl.gov & $208-526-2336$ \\
\hline Craig Primer & $\begin{array}{l}\text { Idaho National } \\
\text { Laboratory }\end{array}$ & $\begin{array}{l}\text { Plant Modernization } \\
\text { Lead }\end{array}$ & Craig.Primer@inl.gov & $208-526-8376$ \\
\hline Jeffrey Joe & $\begin{array}{l}\text { Idaho National } \\
\text { Laboratory }\end{array}$ & $\begin{array}{l}\text { Principal Human } \\
\text { Factors Scientist }\end{array}$ & jeffrey.joe@inl.gov & $208-521-4886$ \\
\hline Jeremy Mohon & $\begin{array}{l}\text { Idaho National } \\
\text { Laboratory }\end{array}$ & $\begin{array}{l}\text { Human Factors } \\
\text { Scientist }\end{array}$ & Jeremy.Mohon@inl.gov & $208-526-1246$ \\
\hline Katya Le BLanc & $\begin{array}{l}\text { Idaho National } \\
\text { Laboratory }\end{array}$ & $\begin{array}{l}\text { Principal Human } \\
\text { Factors Scientist }\end{array}$ & katya.leblanc@inl.gov & $208-526-1395$ \\
\hline Paul Hunton & $\begin{array}{l}\text { Idaho National } \\
\text { Laboratory }\end{array}$ & Research Lead & Paul.Hunton@inl.gov & $208-526-3045$ \\
\hline Rachael Hill & $\begin{array}{l}\text { Idaho National } \\
\text { Laboratory }\end{array}$ & $\begin{array}{l}\text { Human Factors } \\
\text { Scientist }\end{array}$ & Rachael.Hill@inl.gov & $208-526-2259$ \\
\hline Vaibhav Yadav & $\begin{array}{l}\text { Idaho National } \\
\text { Laboratory }\end{array}$ & Research Scientist & Vaibhav.Yadav@inl.gov & $208-526-3910$ \\
\hline $\begin{array}{l}\text { Zachary } \\
\text { Spielman }\end{array}$ & $\begin{array}{l}\text { Idaho National } \\
\text { Laboratory }\end{array}$ & $\begin{array}{l}\text { Human Factors } \\
\text { Scientist }\end{array}$ & Zachary.Spielman@inl.gov & $208-526-1870$ \\
\hline $\begin{array}{l}\text { Asgeir } \\
\text { Droivoldsmo }\end{array}$ & IFE & $\begin{array}{l}\text { Principal Research } \\
\text { Scientist }\end{array}$ & asgeir.droivoldsmo@ife.no & $\mathrm{n} / \mathrm{a}$ \\
\hline Kate Jackson & KeySource & $\begin{array}{l}\text { Director, Energy } \\
\text { and Technology } \\
\text { Consulting }\end{array}$ & $\begin{array}{l}\text { Kathryn.j.jackson@gmail.co } \\
\text { m }\end{array}$ & $412-596-6451$ \\
\hline $\begin{array}{l}\text { Dustin } \\
\text { Greenwood }\end{array}$ & NuScale Power & $\begin{array}{l}\text { Director, Plant } \\
\text { Startup \& Service }\end{array}$ & $\begin{array}{l}\text { dgreenwood@nuscalepowe } \\
\text { r.com }\end{array}$ & $432-978-2231$ \\
\hline Jason Remer & $\begin{array}{l}\text { Remer Consulting } \\
\text { LLC }\end{array}$ & Principal & sjr@remereng.com & $202-431-8204$ \\
\hline Sean Lawrie & ScottMadden & Partner & $\begin{array}{l}\text { seanlawrie@scottmadden.c } \\
\text { om }\end{array}$ & 919-781-4191 \\
\hline $\begin{array}{l}\text { Alison (Krager) } \\
\text { Hahn }\end{array}$ & $\begin{array}{l}\text { U.S. Department of } \\
\text { Energy }\end{array}$ & $\begin{array}{l}\text { Federal Program } \\
\text { Manager }\end{array}$ & $\begin{array}{l}\text { alison.hahn@nuclear.energ } \\
\text { y.gov }\end{array}$ & $301-903-5049$ \\
\hline Clinton Carter & $\begin{array}{l}\text { Utilities Service } \\
\text { Alliance }\end{array}$ & $\begin{array}{l}\text { USA Director - Fleet } \\
\text { Modernization }\end{array}$ & clintoncarter@charter.net & $254-258-3716$ \\
\hline Paul Hippely & $\begin{array}{l}\text { Westinghouse } \\
\text { Electric Company }\end{array}$ & $\begin{array}{l}\text { Manager, Global } \\
\text { I\&C Marketing }\end{array}$ & $\begin{array}{l}\text { hippelpj@westinghouse.co } \\
\text { m }\end{array}$ & 724-994-9449 \\
\hline Charles Edwards & Xcel Energy & $\begin{array}{l}\text { Nuclear } \\
\text { Transformation } \\
\text { Team }\end{array}$ & $\begin{array}{l}\text { charles.edwards@xenuclea } \\
\text { r.com }\end{array}$ & $715-441-8804$ \\
\hline
\end{tabular}




\begin{tabular}{|c|c|c|c|c|}
\hline Name & Company & Title & Email & Phone \\
\hline Don Bosnic & Xcel Energy & $\begin{array}{l}\text { General Manager } \\
\text { Nuclear Fleet } \\
\text { Operations - } \\
\text { Strategy and } \\
\text { Initiatives }\end{array}$ & don.bosnic@xenuclear.com & $315-396-5766$ \\
\hline Gene T. Foote & Xcel Energy & $\begin{array}{l}\text { NPP } \\
\text { Transformation } \\
\text { Team Member }\end{array}$ & gene.foote@xenuclear.com & $612-330-7503$ \\
\hline $\begin{array}{l}\text { Henry H. } \\
\text { Butterworth III }\end{array}$ & Xcel Energy & Project Manager & $\begin{array}{l}\text { henry.butterworth@xenucle } \\
\text { ar.com }\end{array}$ & $715-781-3094$ \\
\hline James Hill & Xcel Energy & $\begin{array}{l}\text { IT Projects Program } \\
\text { Manager }\end{array}$ & james.hill@xenuclear.com & $651-267-7266$ \\
\hline Kyle Frazer & Xcel Energy & $\mathrm{n} / \mathrm{a}$ & $\mathrm{n} / \mathrm{a}$ & $\mathrm{n} / \mathrm{a}$ \\
\hline Marc Anderson & Xcel Energy & $\begin{array}{l}\text { Nuclear Innovation } \\
\text { Engineer }\end{array}$ & $\begin{array}{l}\text { marc.anderson@xenuclear. } \\
\text { com }\end{array}$ & $612-330-6438$ \\
\hline
\end{tabular}




\section{Appendix C}

\section{Example Content for Advanced Capability Pages}

\section{fiduanced Outage Management}

\section{Description:}

Using technology to support the management of refueling outages including: Automated status updates, advanced outage dashboards, advanced outage schedule analysis, remote job oversight, enhanced outage control center designs, and emergent issues resolution.

\section{Capability Benefits}

- Reduced staffing requirements

- Fewer outage extensions

- Tighter Outage schedule control

- Quicker emergent issue resolution

- More effective use of worker's time

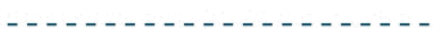

\section{Related Functional Areas:}

- Work Management

- Operations

- Maintenance

- Outage Management

\section{Related Base Technologies:}

- Digital HMI

- WiFi

- Data Integration and Sharing

- Helmet Cameras

- Computer Vision

- EWP/AWP

- Fixed Cameras

- Tablets
LIGHT WATER

REACTOR

SUSTAINABILITY
Applicable Standards, Guidance, and Reports:

- Selected LWRS Reports: INL/EXT14-33182, -16-38265, -16-39622,

$-17-43234$

- IAEA:-TECDOC-1806

\section{Demonstration Resources:}

- Integration of EWP during outage at Palo Verde

- Demonstration of Computer Vision to monitor RCA access at Palo Verde

- Demonstration of enhanced emergent issues management at Palo Verde.

\section{Deployment Resources:}

- Outage Control Center redesign at Brunswick NPP

- Outage Control Center digital upgrade at Palo Verde

- Use of One Note to support outage information at Palo Verde, Sequoyah, South Texas Project.

\section{Point of Contact}

Shawn St Germain 


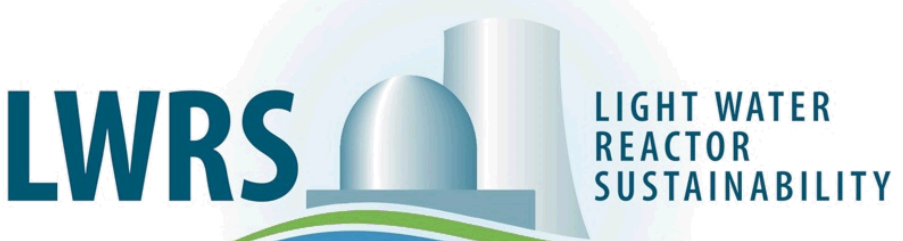

\section{flutomated Rounds}

\section{Description:}

An automated round consists of a set of repeatable monitoring tasks along a specified route performed by an automated platform as opposed to a worker.

\section{Capability Benefits}

- Data Digitalization

- Cost Reduction

- Improved Reliability

- Reduced Workload

- Decreased Risk

- - - - - - - - - - - - - - - - - - - -

Related Functional Areas:

- Operations

- Maintenance Support

- Performance Improvement

- Procedures and Records

\section{Related Base Technologies:}

- Bluetooth

- WiFi

- Machine Learning

- Spatial Mapping

- Computer Vision

- Bar Code Readers

- Mobile Cameras

- GPS Devices

- Drones

- Robots

- RFID

\section{Demonstration Resources:}

- INL-Automation of Data Collection Methods

- International Workshop on the Use of Robotic Technologies at Nuclear Facilities

\section{Deployment Resources:}

- Duke Energy Drone Usage

- Honeywell T\&D Condition Monitoring

- Fukushima Radiation Measurement

\section{Point of Contact}

Ahmad Al-Rashdan

\section{Applicable Standards,} Guidance, and Reports:

- IEEE: 1872,1873

- EPRI: 3002008602,3002003898

- NRC Guidance: NUREG/CP-0304

- Selected LWRS Reports: INL/EXT-18-51456, 17-42918 
LIGHT WATER

REACTOR

SUSTAINABILITY

\section{flutomated Rad. / Cont. Detection}

\section{Description:}

Automated Radiation/ Continuous Detection uses technology to protect employees in nuclear power plants and workplace environments by actively measuring radioactive particulates for safety and environmental purposes.

\section{Capability Benefits}

- Accurate measurement of radiation

- Early warning system for workers

- Increased worker efficiency

- Optimize safety and operational efficiency

- Reduce dependence on human decision making

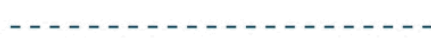

\section{Related Functional Areas:}

- Radiation Protection

- Operations

- Maintenance

- Emergency Planning

- Performance improvement

- Regulatory services

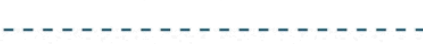

\section{Related Base Technologies:}

- Wireless Sensors

- Wired Sensors

- Digital I\&C

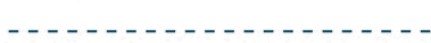

Applicable Standards, Guidance, and Reports:

- Selected LWRS Reports: INL/EXT18-52206, -18-51457, -18-51456
Demonstration Resources:

- Lighthouse directional radiation detectors at Los Alamos National Laboratory

- Superconducting Tunnel Junction detectors at Lawrence Livermore National Laboratory.

\section{Deployment Resources:}

- Use of innovative "lighthouse" detectors in Los Alamos National Laboratory

- Roadside Tracker at Oakridge National Laboratory detects radiation sources in vehicles.

Point of Contact

Shawn St Germain 


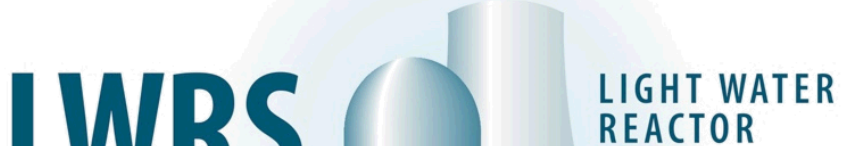 \\ REACTOR \\ SUSTAINABILITY}

\section{futomated Chemical Sampling}

\section{Description:}

Automated chemical sampling describes the ability to safely and reliably sample a chemical process line without the need of an operator or operator supervision.

\section{Capability Benefits}

- Improves process safety

- Increases operator safety

- Reduces operator workload

- Improves operational efficiency

- Increases sample homogeneity

- Potential for automated laboratory analysis
Related Functional Areas:

- Operations

- Regulatory Services

- Quality Control

- Chemistry

- Radiation Protection

- Instrumentation and Controls (I\&C)

- Contamination Protection

- Contamination Protection

Related Base Technologies:

- Process equipment

- Wired/Wireless Sensors

- WiFi

- LoRa

- $900 \mathrm{MHz}$

- Data Integration and Sharing

- Anomaly Detection
Applicable Standards, Guidance, and Reports:

- EPRI: 3002010645,3002000505

- ISO: 3165:1976, 2889:2010

\section{Demonstration Resources:}

- In-Line Automated Sampling Coupled with Laboratory Analysis - 2018 Smart Chemistry Demo

\section{Deployment Resources:}

- Example of proven technology Sentry In-line Automatic Samplers

\section{Point of Contact}

Shawn St Germain 
LIGHT WATER

REACTOR

SUSTAINABILITY

\section{futomated Technical Specification Monitoring}

\section{Description:}

Automated technical specification (TS) monitoring is the process by which limiting conditions for operation are actively monitored remotely to update operations staff on the status of a plant.

\section{Capability Benefits}

- Expediated Event Response

- Assistance in Monitoring for Emergency Events

- Enhanced Human Performance

- Enhanced Event Pattern Recognition

- Early Detection of Potentially Operation Limiting Events

Related Functional Areas:

- Regulatory Services

\section{Related Base Technologies:}

- $4 / 5 \mathrm{G}$

- WiFi

- Machine Learning

- Data Integration and Sharing

- Anomaly Detection

- Wireless Sensors

- Digital I\&C

Applicable Standards, Guidance, and Reports:

- IEEE: 1249,758

- EPRI: 1006833,1022988

- NRC Guidance: NUREG/CR-6895

- Selected LWRS Reports: INL/EXT12-27754, -11-24154

\section{Demonstration Resources:}

- IAEA: Automation of Technical Specification Monitoring for Nuclear Power Plants

Deployment Resources:

- Automated Real-Time Monitoring of Hydrological Parameters

- Automated Tech Spec Monitoring at the Callaway Plant Site

\section{Point of Contact}

Vivek Agarwal 
LIGHT WATER

REACTOR

SUSTAINABILITY

\section{flutomated Work Packages [AWP]}

\section{Description:}

Improvement of current activities using data sources intelligently to drives work processes in an effort to migrate plants from manual maneuvers to a novel data-driven systematic approach.

\section{Capability Benefits}

- Automated Work processes

- Enhance Human Performance

- Lower Material Demand

- Reduce human error

- Process Efficiency

- Lower Labor

\section{Related Functional Areas:}

- Procedures and Records

- System Engineering

- Work Management

- Design Engineering

- Operations

- Maintenance

Example Content
Related Base Technologies:

- Machine Learning

- Data Storage

- Data Integration and Sharing

- NLP/Text Mining

\section{Applicable Standards,} Guidance, and Reports:

- Object Maintenance Group: UML

- Selected LWRS Reports: INL/EXT16-38809, -17-43264, -18-51456,

$-18-44497,-18-44972$

\section{Demonstration Resources:}

- Electronic Shift Operations Management System (eSOMS) Clearance Tag Sharing Overview, ABB, 2015

- EPRI: Use of LTE Cellular Network and Distributed Antenna Systems to Improve Connectivity and Increase Data Transfer: A Plant Monitoring Initiative

- Operations and Maintenance Development: Work Planning Assessment Guidelines for Nuclear Power Plant Personnel, Electric Power Research Institute

\section{Deployment Resources:}

- Perpetua announces an Energy Harvester for use with Honeywell XYR6000 One Wireless transmitters

\section{Point of Contact}

Ahmad Al Rashdan 


\section{LWRS @}

\section{Computer Based Procedures}

\section{Description:}

A computer based procedure (CBP) is defined as a dynamic presentation of a procedure that guides the user seamlessly through the logical sequence of the procedure.

\section{Capability Benefits}

- Automatic place-keeping

- Ease of navigating between and within procedures

- Enhanced human performance

- Reduced Workload

- Improved operations and maintenance efficiency.

\section{Related Functional Areas:}

- Operations

- Procedures and Records

- Maintenance Support

- Work Management

\section{Related Base Technologies:}

- 4G

- WiFi

- Tablets

- Bar Code Readers

- RFID

- Wireless Sensors

- Mobile Cameras

- Data Storage

- Data Integration and Sharing

Applicable Standards, Guidance, and Reports:

- IEEE: 1786

- EPRI: 3002004310

- NRC Guidance: NUREG-6634, $-0700,-0711,-0800$

- Selected LWRS Reports: INL/EXT15-35284
Demonstration Resources:

- LWRS: INL-PVGS Collaboration

\section{Deployment Resources:}

- IAEA: Dynamic computer based procedures system for the AP1000 plant

\section{Point of Contact}

Katya Le Blanc 
LIGHT WATER

REACTOR

SUSTAINABILITY

\section{Computer Operated Support System}

\section{Description:}

The computerized operator support system (COSS) supports operators in cognitive activities such as situation assessment and response planning by using digital technology that provide recommendations and warnings.

\section{Capability Benefits}

- Improved process safety

- Improved operational efficiency

- Reduced workload

- Enhanced human performance and situation awareness

- Stream-lined and informed decision making

\section{Related Functional Areas:}

- Operations

- Instrumentation and Controls (I\&C)

- Outage Management

- Emergency Planning

\section{Related Base Technologies:}

- Data Integration and Sharing

- Data Storage

- Anomaly Detection

- Artificial Intelligence

- Machine Learning

- Digital I\&C

- Digital HMI
Demonstration Resources:

- LWRS: INL-PVGS Collaboration

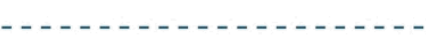

Deployment Resources:

- INL/CON-14-32523: Simulated Control Room with COSS

Point of Contact

Ronald L. Boring

Applicable Standards,

Guidance, and Reports:

- EPRI: 1000804,1004674

- ISO: 10303-240

- Selected LWRS Reports: INL-EXT-12-275205, -17-43390

- NRC Guidance: NUREG-0700 


\section{LWRS@D}

\section{Remote Supervision}

\section{Description:}

Remote supervision is the ability of a manager or specialist to supervise worker interactions and work processes remotely in real-time.

\section{Capability Benefits}

- Reduced Risk

- Enhanced Human Performance

- Enhanced Worker Confidence

- Improved operations and maintenance efficiency.

- Worker Status Verification

\section{Related Base Technologies:}

- 4/5G

- WiFi

- Virtual Reality

- Augmented Reality

- Wireless Sensors

- Mobile Cameras

- Recording

- Tablets

- Helmet Cameras

- HUD Headsets

\section{Deployment Resources:}

- EPRI: 3002005480

Point of Contact

Ahmad Al-Rashdan

\section{Applicable Standards,}

Related Functional Areas:

- Operations

- Training Guidance, and Reports:

- Work Management

- Functional Area Management

- Maintenance support

- Project Management

- IEEE: C37.1

- EPRI: 1014533,3002005480 3002001131

- NRC Guidance: NUREG-1556, NUREG/CR-6751

- Selected LWRS Reports: INL/EXT16-39098, 18-51457 


\section{Example Content for Base Technology Pages}

\section{Digital Human Machine Interface}

\section{Description:}

The human-machine interface (HMI), also referred to as humansystem interface $(\mathrm{HSI})$, is part of the system through which plant personnel (e.g., operators) interact to perform their functions and tasks. Machine/system refers to a nuclear power plant.

Applicable Advanced Capabilities/Integrated Technologies:

- Digital Control Room (DCR)

- coss

- DCS

- Remote Supervision

\section{Available Vendors:}

- Westinghouse/ Ovation

- General Electric (GE)

- Honeywell Experion

- Mitsubishi

- Curtiss-Wright

Example Content
Utilities Who Have Implemented or Will Implement the Technology:

- Palo Verde

- Southern

- Duke

- Dominion

\section{Applicable Guidelines and} Standards for Technology Implementation:

- Regulatory Standards: 10 CFR 50.59

- NRC Guidance: NUREG-0700, $-0711,-0737,-0800$

- ISO Guidance: ISO 9241

- EPRI Guidance: 3002004310 3002011816
LIGHT WATER

REACTOR

SUSTAINABILITY
Implementation Suggestions:

To ensure successful

implementation of digital HMIs, it is highly suggested to fully involve human factors engineering into the development of the design and functionality of digital HMIs that control various plant functions. Additional reading:

- INL/EXT-18-51107

- INL/EXT-18-44798

- INL/EXT-18-51212

- INL/EXT-18-51365 
LIGHT WATER

REACTOR

SUSTAINABILITY

\section{Matural Language Processing}

\section{Description:}

Natural language processing (NLP) is a branch of artificial intelligence that helps computers understand, interpret, and manipulate human language. One application of NLP in nuclear is to extract information from commonly used plant documents such as procedures.

\section{Applicable Advanced} Capabilities/Integrated Technologies:

- Automated Work Packages

- Advanced Outage Management

\section{Available Software:}

- IBM Watson Tone Analyzer

- Amazon Comprehend

- Google Cloud Natural Language

- Apache openNLP

- PyNLPI

- Atos

\section{Suggested Reading:}

- Selected LWRS Reports: INL/EXT-18-51474 
LIGHT WATER

REACTOR

SUSTAINABILITY

\section{Data Integration and Sharing}

\section{Description:}

Data integration and sharing combines technical and business processes to combine data from different sources into meaningful and valuable information.

Applicable Advanced Capabilities/Integrated Technologies:

- EWP/AWP

- Computer Based Procedures

- coss

- Advanced Outage Management

- Automated Technical Specification Monitoring

- Automated Chemical Sampling
Utilities Who Have

Demonstrated the Technology:

- Palo Verde

- Cooper

- Xcel Energy

- Exelon

- Southern

Suggested Reading:

- Selected LWRS Reports:

INL/EXT-17-42918

INL/EXT-19-54294 
LIGHT WATER

REACTOR

SUSTAINABILITY

\section{Artificial Intelligence}

\section{Description:}

Artificial Intelligence (Al) is the ability of a computer or other machine to perform actions thought to require intelligence.

Applicable Advanced Capabilities/Integrated Technologies:

- Computer Operated Support System

- Condition Based Monitoring

- Advanced Outage Management

- Automated Detection

- Automated Sampling
Industries and Organizations

Researching or Applying Al:

- Universities

- National Labs

- Siemens

- Institute for Energy Technology

- General Electric (GE)

- Beyond Limits (Oil \& Gas, NASA)

\section{Applicable Guidelines and} Standards for Technology Implementation:

- NRC Guidance: NUREG/CR-68 12 \& ORNL/TM-2003/22 


\section{Appendix E}

\section{Tools Applicable to Nuclear Innovation}

\section{Completing Functional Analysis}

\section{Description:}

Functional Requirements Analysis (FRA) identifies functions that are needed to meet plant safety objectives. FRA entails functional allocation (FA) where identified functions are assigned to a level of automation (automatic, shared, manual).

\section{Important elements to the functional analysis:}

Successful FRA and FA account for a thorough set of inputs to ensure optimized allocation.

\section{Suggested reading:}

- LWRS Reports: INL/EXT-17-42190, $-17-43226$

- EPRI: 3002004310,3002011816

- NRC: NUREG-0700, $-0711,-0800$

- Hollnagel, E., \& Bye, A. (2000). Principles for modelling function allocation. International Journal of Human-Computer Studies, 52(2), 253-265.

- Feigh, K. M., \& Pritchett, A. R. (2014). Requirements for effective function allocation: A critical review. Journal of cognitive engineering and decision making, 8(1), 23-32.

LIGHT WATER

REACTOR

SUSTAINABILITY

\section{SELECTED \\ FUNCTION \\ Should this function be automated?

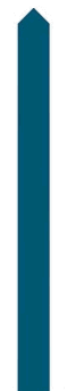

\begin{tabular}{|c|c|c|c|}
\hline Technical Feasibility & Regulatory Requirements & Safety \& Performance & Other Input \\
\hline Complexity/Feasibititiy & 10CFR50.59 & Automation Capabilities & Personnel Preferences \\
\hline Costs & 10CFR50.34 & Human Capabilities & OER Findings \\
\hline Development Time & 10CFR52.47 & HFE Principles \& Guidelines & Existing practices \\
\hline
\end{tabular}

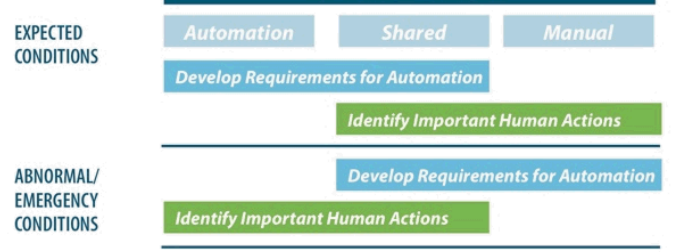


LIGHT WATER

REACTOR

SUSTAINABILITY

\section{Performing Task fnalysis}

\section{Description:}

Task analysis is a broad category of methods used to systematically document the human interactions with a system. Task analysis in innovation is used to inform the selection of new technologies or processes to enhance humansystem interaction.

\section{Important steps in performing task analysis:}

There are many variants of task analysis, each with their own benefits. However, task analysis can be broadly performed from the following process in an iterative fashion.

\section{1: Define Scope of the Task Analysis}

- Focus on tasks that are only pertinent to the new system or modification.

- Identify relevant input (e.g., OER) from other activities.

- Clearly define who the end users are.

- Schedule time to meet end users to collect task data.

\section{2: Collect Task Data}

- Perform observations, verbal protocol, talk/walk-throughs, to learn how tasks are accomplished.
- Integrate findings with other task analysis inputs like existing procedures, OER findings, and/or prior task analysis results.

\section{3: Interpret Collected Data}

- Task data is interpreted as a hierarchical illustration, detailed description, or sequence diagram.

- Hierarchical task analysis (HTA) is goal-oriented and visually breaks a task into sub-tasks in a top-down hierarchical format.

- Tabular task analysis (TTA) allows for detailed analysis of potential failure modes for each important human action.

- Link analysis (LA) and operational sequence diagrams (OSD) describe the sequence of actions with corresponding system elements.

\section{4: Develop Innovation Solutions}

- While some design insights are gleaned immediately after simply talking to users, many insights are not formulated until careful inspection of the task analysis results. HTA and TTA can identify human actions that may be best supported with either new technology or processes to support a specific functional area. LA and OSD can identify optimal groupings of the elements needed to support a process, task, or function.

\section{Suggested reading:}

- EPRI: 3002004310

- NRC: NUREG-0711

- Kirwan, B., \& Ainsworth, L. K. (1992). A guide to task analysis: the task analysis working group. CRC press.

- Stanton, N. A., Salmon, P. M., Rafferty, L. A., Walker, G. H., Baber C., \& Jenkins, D. P. (2017). Human factors methods: a practical guide for engineering and design. CRC Press.

\section{Outcomes of task analysis:}

- Systematic description of the actions each end user must do to accomplish a task.

- Provides a formal technical design basis for certain design decisions that ensure the end users' physical and cognitive capabilities are considered. 
LIGHT WATER

REACTOR

SUSTAINABILITY

\section{Gathering Requirements}

\section{Process}

- Identify the drivers for project

- What problem are we solving?

- How can we meet those needs while causing the least amount of headache and disruption to affected parties?

- How can we meet those needs while making everyone's job a little easier

- Clearly identify the goals of the project

- What would success look like?

- Frame success in terms of the business need

- Identify the people, processes, and systems that connect to the new system

- A user interacts directly with the system

- A stakeholder is affected by it

- Ask what us and stakeholders need and want

- Prioritize needs based on alignment with business goals

- Anticipate unintended consequences and mitigate them

- How can we meet those needs while causing the least amount of headache and disruption to affected parties?

- How can we meet those needs while making everyone's job a little easier

\section{Common Challenges and Tips}

- Over represent minor stakeholders

- Identify who and what is most affected by the system on a daily basis, prioritize these users

- Management and supervisors are often asked for input, ask the boots on the ground, they be the most affected and will have the most insight

- Leave out whole groups of users

- Look at org charts to identify hidden users

- Solve one problem and introduce another

- Ask for users to imagine a bad system. How would it function?

- Carry over unnecessary legacy requirements from old process to new system

- Critically evaluate requirements

- Review requirements against business needs

- Does this accomplish the objective?

- Does this solve one problem and introduce another

- Be open to feedback and always ask how things could go wrong 
LIGHT WATER

REACTOR

SUSTAINABILITY

\section{f Human-Centered Approach}

\section{Description:}

In order for implementation to be effective, it needs to be designed with the end user in mind. Without knowing your users, their tasks, and their environment, the final product is at significant risk for failure.

A human-centered approach consists of three fundamental elements:

An effective human-centered approach requires understanding your user's needs, designing solutions to fit these needs, and then evaluating the efficacy of the designed solutions. This process is iterative where each subsequent phase builds upon lessons learned from the previous design.

Putting the greatest resources in earlier understanding activities is most critical in ensuring that new innovation comprehensively meets users' needs.
Outcomes of a humancentered approach:

- Identification and mitigation of critical problems in the technology earlier on, enabling a cost-effective approach to innovation.

- 'Buy-in' to the technology across all organizational levels (topdown and bottom-up).

\section{Common mistakes:}

- Focusing on only a subset of user types when gathering requirements.

- Making assumptions that certain features or functions are useful or needed without vetting with the end user.

- Failing to get early design feedback when modifications are most feasible.

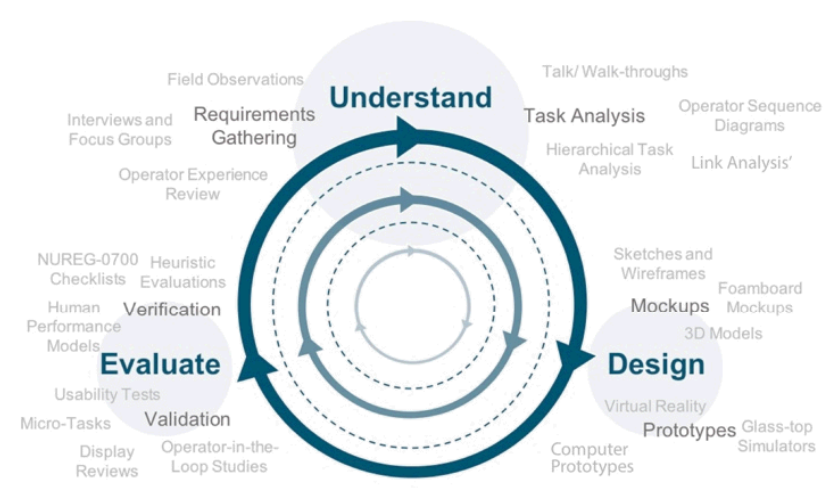


LIGHT WATER

REACTOR

SUSTAINABILITY

\section{Designing for User Meeds}

The role of design goes beyond product innovation. Innovation programs and processes also benefit from design activities. Design helps refine concepts during implementation by providing a way for end users to provide feedback based on their needs.

\section{Designing for user needs:}

Iterative designs that are vetted regularly by the end users is a key recipe for success.

\section{Outcomes of design:}

- Focused representations (i.e., prototypes) of the actual capabilities that can be used for early evaluations (verification and validation activities).

- When done correctly (iteratively), each prototype documents the incremental improvements to the overall concept, which is essential for benchmarking.

\section{Suggested reading:}

- LWRS Reports: INL/EXT-18-51107, INL/EXT16-38576

- EPRI: 3002004310

- Rothwell, R. (1992). Successful industrial innovation: critical factors for the 1990s. R\&D Management, 22(3), 221-240.

- Snyder, C. (2003). Paper prototyping: The fast and easy way to design and refine user interfaces. Morgan Kaufmann.

- Gothelf, J. (2013). Lean UX: Applying lean principles to improve user experience." O'Reilly Media, Inc.".

\section{ENTER DESIGN}

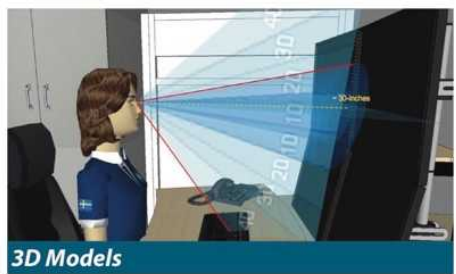

Foam mockups and 30 Models can be rapidily developed to compare different concepts bassed on their impact to ergonamics, looded in VR for an immersive walkhthough

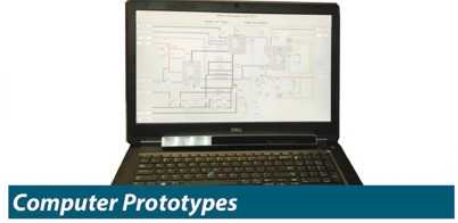

Foam mockups and 30 Models can be rapidly developed to compare different concepts based on their impact to ergonamics, loaded in VR for an immersive walkthrough.

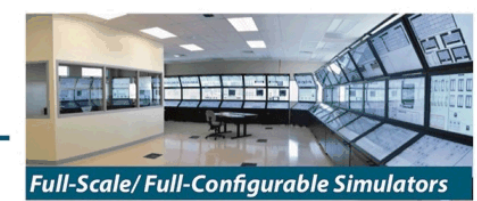

Common Software Tools:

Wireframing: Visio, PowerPoint, Evolus Pencil, Adobe

Illustrator

Interactive: Axure, Sketch, Adobe XD, SketchFlow

30 Models: SketchUp

Languages: (\#
Simulators can be used to holistically validate the benefits of advanced control room prior to final implementation in a training smulator or actual control room. 


\section{LWRS A}

Ualidating Designs with Users

\section{Description:}

Validation of new concepts should be done early and often with end users. It is important to capture both user preferences and human-system performance to ensure concepts meet their requirements.

\section{Important elements of validating new concepts with users:}

Successful validation of concepts should be goal-oriented with focus made specific to a design question or requirement at hand. Because one method or measure cannot adequately validate a concept, using a strategic suite of methods and measures is important.

\section{Outcomes of design:}

- Focused representations (i.e., prototypes) of the actual capabilities that can be used for early evaluations (verification and validation activities).

- When done correctly (iteratively), each prototype documents the incremental improvements to the overall concept, which is essential for benchmarking.

\section{Suggested reading:}

- LWRS Reports: INL/EXT-15-36704, $-17-43250,-18-51107$

- EPRI: 3002004310

- Kovesdi, C., Joe, J., \& Boring, R. (2018). A guide for selecting appropriate human factors

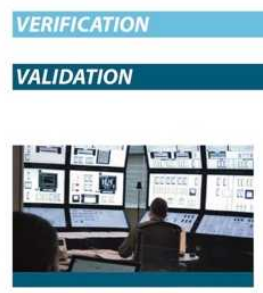

Researcher scoring human-system performance from observation deck.

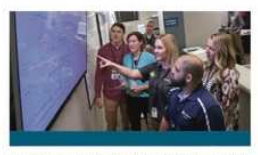

Operators providing feed backin display review

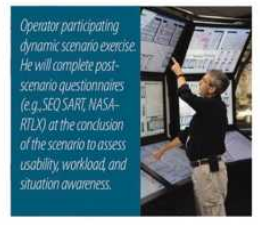

methods and measures in control room modernization efforts in nuclear power plants. In International Conference on Applied Human Factors and Ergonomics (pp. 441-452). Springer, Cham. 
LIGHT WATER

REACTOR

SUSTAINABILITY

\section{Uerifying Concepts using Principles}

\section{Description:}

Verification is the process of evaluating a design or concept to determine whether it provides the information and functionality needed to accomplish a task and whether it conforms to human factors engineering (HFE) guidance and design principles.

\section{Important elements to the verification process:}

Verification is an essential activity in concept evaluation to determine if sound engineering and design principles are reflected in the design.

\section{Suggested reading:}

- LWRS Reports: INL/EXT-12-26787, INL/EXT-16-38576

- EPRI: 3002004310

- NRC: NUREG-0700,-0711, -0800

- Stanton, N. A., Salmon, P. M., Rafferty, L. A., Walker, G. H., Baber, C., \& Jenkins, D. P. (2017). Human factors methods: a practical guide for engineering and design. CRC Press.

\section{Outcomes of verification:}

- Consistent design language applied across all vendor-specific technologies.

- Assurance that sound engineering and design principles are reflected.

- Traceability of specifications to industry-accepted standards and principles.

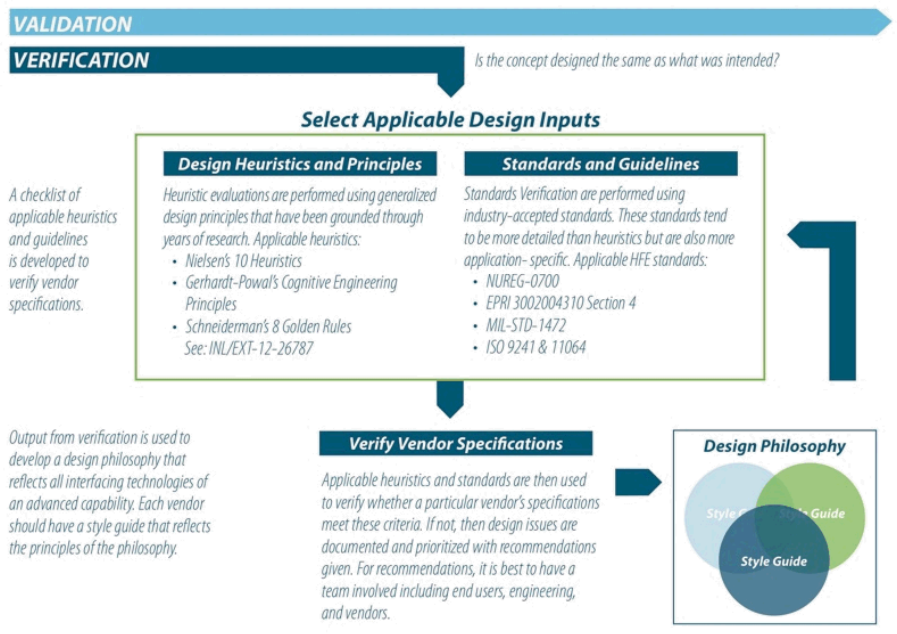




\section{Appendix F}

\section{Facilitated Discussion Semi-Structured Questions}

\begin{tabular}{|c|c|}
\hline $\begin{array}{l}\text { Phase 1: } \\
\text { Identify }\end{array}$ & 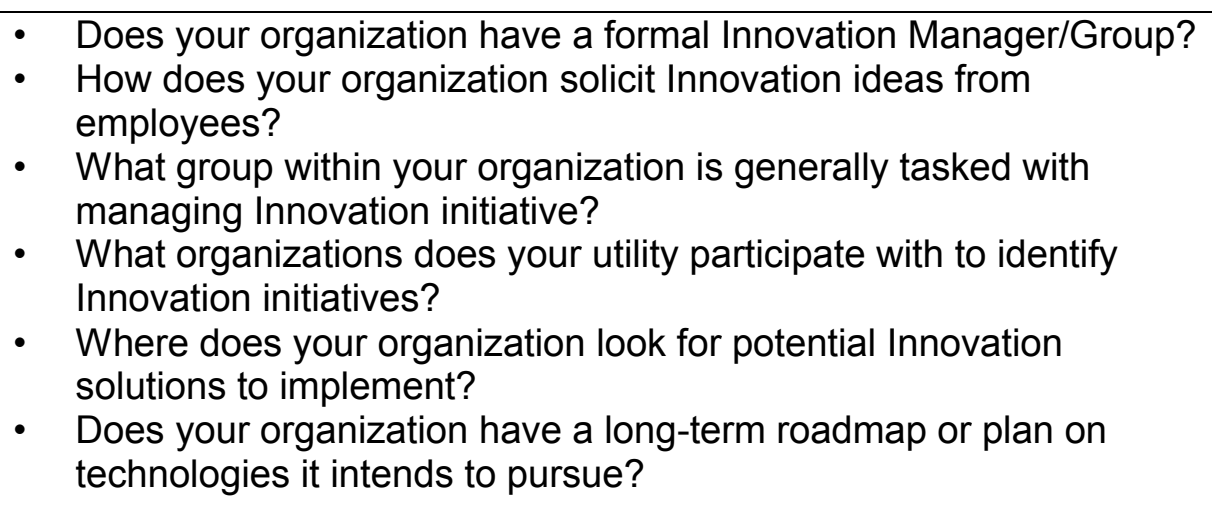 \\
\hline $\begin{array}{l}\text { Phase 2: } \\
\text { Select }\end{array}$ & $\begin{array}{l}\text { - How do you do you gather and evaluate requirements? } \\
\text { - How do you document requirements? } \\
\text { - } \quad \text { requirements at each stage? } \\
\text { Is there a project that you have missed important requirements } \\
\text { that ultimately affected the success of the project? } \\
\text { - How could you have captured those requirements earlier? } \\
\text { - Have you changed your process to ensure you don't miss } \\
\quad \text { lesso there subsequent projects that have benefitted from the } \\
\text { - Do you have a formal process for evaluating technologies? } \\
\text { When evaluating and comparing potential projects how do you } \\
\text { compare the cost/benefit? Are there certain factors that matter } \\
\text { more than others? } \\
\text { When comparing different technologies for the same project, how } \\
\text { do you compare the cost/benefit of each technology, are the } \\
\text { factors different than above? } \\
\text { Do you revisit assumptions about costs and benefits throughout } \\
\text { the project? } \\
\text { - How do you evaluate risk? } \\
\text { - Do you have a defined threshold of acceptable risk? } \\
\text { - What is it based on (e.g., cost, schedule, project success, } \\
\text { - or a combination)? } \\
\text { Who has input into the selection process for choosing a project? } \\
\text { specific project? }\end{array}$ \\
\hline
\end{tabular}




\begin{tabular}{|c|c|}
\hline $\begin{array}{l}\text { Phase 3: } \\
\text { Implement }\end{array}$ & 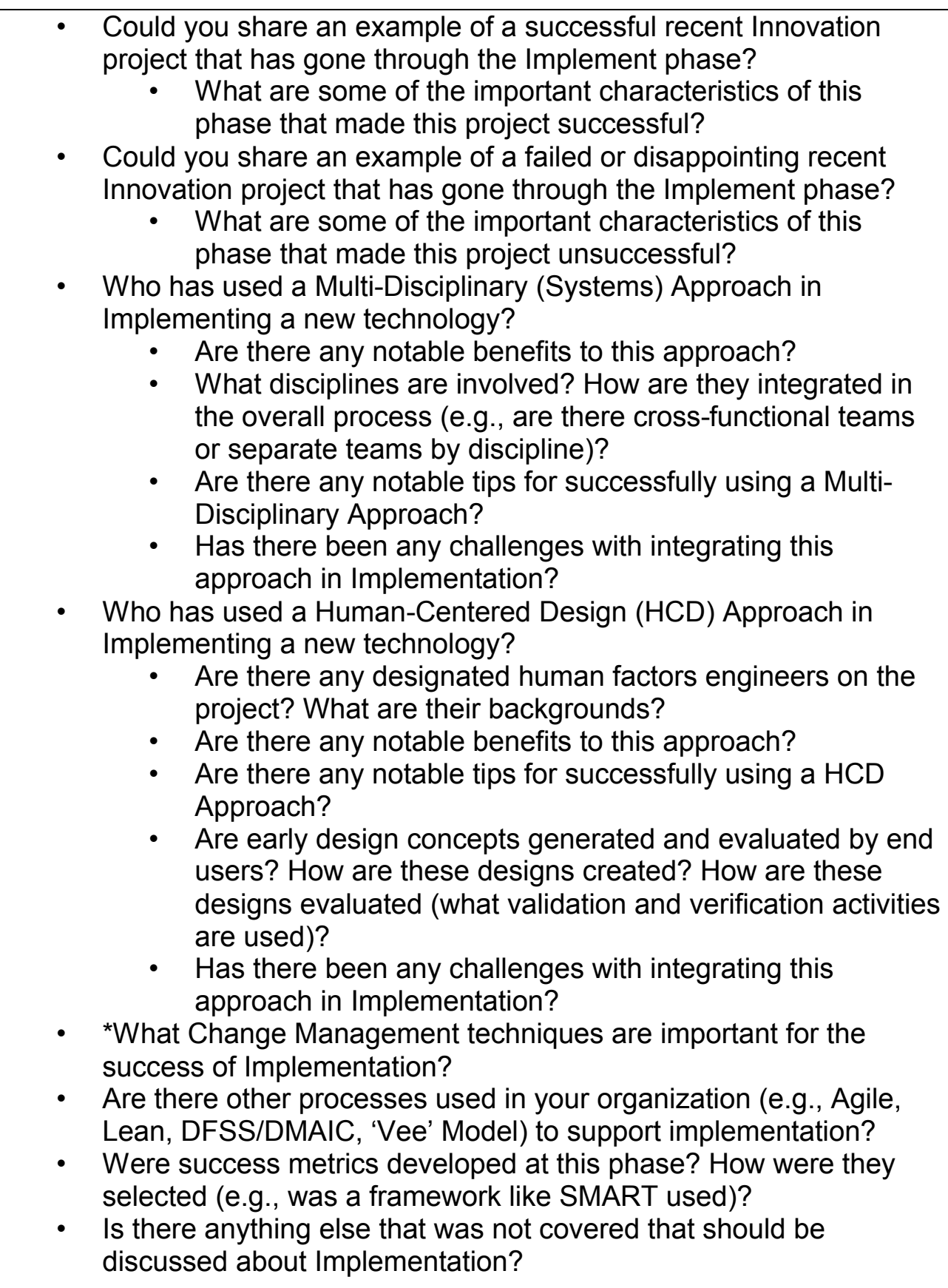 \\
\hline $\begin{array}{l}\text { Phase 4: } \\
\text { Evaluate }\end{array}$ & NA (Session was comprised of freeform discussion) \\
\hline
\end{tabular}




\section{Appendix G}

\section{Presenter Biographies}

\begin{tabular}{|c|c|}
\hline Presenter (Phase) & Iy \\
\hline $\begin{array}{l}\text { Dustin Greenwood } \\
\text { Director of Plant Startup } \\
\text { and Services at NuScale } \\
\text { (Phase 1: Identify) }\end{array}$ & $\begin{array}{l}\text { Director Plant Startup and Services is a diversely experienced professional with } 25 \\
\text { years of experience in design, construction, and operations of nuclear facilities. Dustin } \\
\text { is responsible for the development and implementation of NuScale services and for } \\
\text { startup has developed the Initial Testing Program (ITP) and the Inspections, Tests, } \\
\text { Analysis, and Acceptance Criteria (ITAAC). In addition, he is responsible for the basis } \\
\text { of the plant staffing levels and costs required for all regulatory required Operations } \\
\text { and Maintenance (O\&M) programs. Most recently Dustin was the Operations Manager } \\
\text { of a uranium enrichment facility and before that a licensed Control Room Supervisor } \\
\text { (CRS) at Energy Northwest. }\end{array}$ \\
\hline $\begin{array}{l}\text { Patrick Kopfle } \\
\text { Nuclear Technology \& } \\
\text { Innovation Consultant at } \\
\text { Dominion Energy } \\
\text { (Phase 2: Select) }\end{array}$ & $\begin{array}{l}\text { Nuclear Technology \& Innovation Consultant at Dominion Energy is responsible for } \\
\text { innovation projects and application of emerging technologies in Dominion Energy's } \\
\text { nuclear plants. He previously worked at Westinghouse Electric Company in AP1000 } \\
\text { design/project development and helped lead the initial deployment of the new plant } \\
\text { innovation programs. Patrick has a Bachelor of Science in Mechanical Engineering } \\
\text { from Virginia Tech and an MBA from the University of Pittsburgh. }\end{array}$ \\
\hline $\begin{array}{l}\text { James Hill } \\
\text { IT Projects Program } \\
\text { Manager at Xcel Energy } \\
\text { (Phase 3: Implement) }\end{array}$ & $\begin{array}{l}\begin{array}{l}\text { Jim Hill is a Senior applications Delivery (IT) Manager } \\
\text { with Xcel Energy. His nuclear career has spanned from } \\
\text { a hands-on machinist mate and radiation-chemistry } \\
\text { specialist in the US nuclear navy to various Engineering, } \\
\text { Quality, Performance Improvement, and Project } \\
\text { management positions in plant and corporate } \\
\text { management roles. His current focus is to improve plant } \\
\text { operation through the use of innovative IT applications } \\
\text { and infrastructure. He has an electrical engineering } \\
\text { degree, an MBA, is a licensed Professional Engineer, } \\
\text { and has held a Senior Reactor Operator license, issued } \\
\text { by the NRC. } \\
\text { He is a Senior Member of the American Society of }\end{array} \\
\text { Quality. His certifications include Manager of Quality/Organizational Excellence, } \\
\text { Project Management Professional, and ITIL Foundation. He has served with the } \\
\text { Midwest-based Performance Excellence Network as an Evaluation Team Leader and } \\
\text { Chief Judge in the Baldrige Performance Excellence evaluation process, used by } \\
\text { organizations to assess and improve their performance. He is a past member of the } \\
\text { Nuclear Energy Institute NEI) Standard Nuclear Performance Model working group, } \\
\text { and has also consulted and contracted with International Atomic Energy Association } \\
\text { (IAEA) working groups for nuclear Knowledge Management guides. He is the current } \\
\text { Chair of the Infrastructure \& Applications subcommittee of the Nuclear Strategic IT } \\
\text { Leadership group (NITSL). }\end{array}$ \\
\hline $\begin{array}{l}\text { Asgeir Drøivoldsmo } \\
\text { Principal Research } \\
\text { Scientist at the Institute } \\
\text { for Energy Technology } \\
\text { (Phase 4: Evaluate) }\end{array}$ & $\begin{array}{l}\text { Current position: Principal research scientist, Human Centered Digitalization, Institute } \\
\text { for Energy Technology, Norway. } \\
\text { He has been working with development and implementation of new operation } \\
\text { concepts, technology and safety in nuclear and petroleum industrial research for more } \\
\text { than } 20 \text { years. The focus in his work has been within practical methodologies based } \\
\text { on principles from the industrial psychology, and he has been working in the } \\
\text { intersection between research and implementation of technology and new work } \\
\text { practices. He holds a BS in computer science, MS degree in cognitive psychology and } \\
\text { a PhD in industrial psychology from the Norwegian University of Science and } \\
\text { Technology (NTNU). }\end{array}$ \\
\hline
\end{tabular}




\section{Appendix $\mathrm{H}$}

\section{Post-Workshop Feedback Survey}

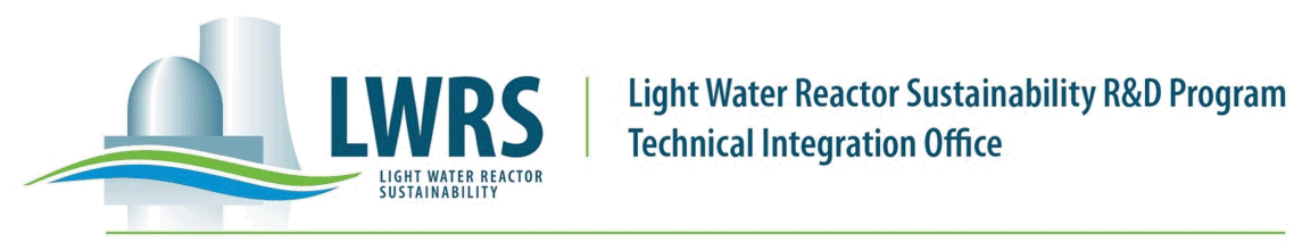

Light Water Reactor Sustainability Program Nuclear Innovation Workshop Feedback Survey

Instructions: Please provide feedback regarding the LWRS Nuclear Innovation Workshop.

1. Overall, how would you rate the workshop?

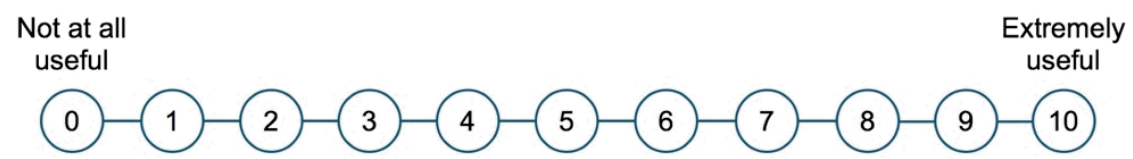

2. What did you like about the workshop?

3. What did you dislike about the workshop?

4. What topics were most useful for you at this workshop? 
5. Would you be interested in participating in an Innovation Working Group if established?

$$
\text { Yes No }
$$

6. If 'Yes' was marked above (Question 5), please provide your contact information below:

Name:

Company/Org:

Email:

7. Would an Innovation Portal to support innovation in the US nuclear industry be useful?

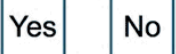

8. Is there anything else you'd like to share about the workshop? 